Research Article

\title{
On the Theory of Fractional Calculus in the Pettis-Function Spaces
}

\author{
Hussein A. H. Salem \\ Department of Mathematics and Computer Science, Faculty of Science, Alexandria University, Alexandria, Egypt \\ Correspondence should be addressed to Hussein A. H. Salem; hssdina@alex-sci.edu.eg
}

Received 19 February 2018; Accepted 2 April 2018; Published 15 May 2018

Academic Editor: Gestur Ólafsson

Copyright (c) 2018 Hussein A. H. Salem. This is an open access article distributed under the Creative Commons Attribution License, which permits unrestricted use, distribution, and reproduction in any medium, provided the original work is properly cited.

\begin{abstract}
Throughout this paper, we outline some aspects of fractional calculus in Banach spaces. Some examples are demonstrated. In our investigations, the integrals and the derivatives are understood as Pettis integrals and the corresponding derivatives. Our results here extended all previous contributions in this context and therefore are new. To encompass the full scope of our paper, we show that a weakly continuous solution of a fractional order integral equation, which is modeled off some fractional order boundary value problem (where the derivatives are taken in the usual definition of the Caputo fractional weak derivative), may not solve the problem.
\end{abstract}

\section{Introduction and Preliminaries}

The issue of fractional calculus for the functions that take values in Banach space where the integrals and the derivatives are understood as Pettis integrals and the corresponding derivatives has been studied for the first time by the authors of $[1,2]$. Following the appearance of [1], there has been a significant interest in the study of this topic (see, e.g., [3-6]; see also [7-9]). This paper is devoted to presenting general results and examples for the existence of the fractional integral (and corresponding fractional differential) operators in arbitrary Banach space where it is endowed with its weak topology. In our investigations, we show that the well-known properties of the fractional calculus for functions taking values in finite dimensional spaces also hold in infinite dimensional spaces. Our results extend all previous contributions of the same type in the Bochner integrability setting and in the Pettis integrability one.

For the readers convenience, here we present some notations and the main properties for the Pettis integrals. For further background, unexplained terminology and details pertaining to this paper can be found in Diestel et al. $[10,11]$ and Pettis [12].

Throughout this paper, we consider the measure space $(I, \Omega, \mu)$, where $I=[0,1], 0 \leq a<b<\infty$ denote a fixed interval of the real line, $\Omega$ denotes the Lebesgue $\sigma$-algebra $\mathscr{L}(I)$, and $\mu$ stands for the Lebesgue measure. $E$ denotes a real Banach space with a norm $\|\cdot\|$ and $E^{*}$ is its dual. By $E_{\omega}$ we denotes the space $E$ when endowed with the weak topology generated by the continuous linear functionals on $E$. We will let $C\left[I, E_{\omega}\right]$ denote the Banach space of weakly continuous functions $I \rightarrow E$, with the topology of weak uniform convergence. And $P[I, E]$ denotes the space of $E$ valued Pettis integrable functions in the interval $I$ (see $[10,12]$ for the definition). Recall that (see, e.g., [10,13-19]) the weakly measurable function $x: I \rightarrow E$ is said to be Dunford (or Gelfand) integrable on $I$ if and only if $\varphi x$ is Lebesgue integrable on $I$ for each $\varphi \in E^{*}$.

Definition 1. Let $p \in[1, \infty]$. Define $\mathscr{H}^{p}(E)$ to be the class of all weakly measurable functions $x: I \rightarrow E$ having $\varphi x \in L_{p}(I)$ for every $\varphi \in E^{*}$.

If $p=\infty$, the added condition

$$
\text { l.u.b. } \|_{\| \varphi=1}(\underset{t \in I}{\operatorname{ess} \sup }|\varphi x(t)|)<\infty
$$

must be satisfied by each $x \in \mathscr{H}^{\infty}(E)$. We also define the class $\mathscr{H}_{0}^{p}(E)$ by

$$
\mathscr{H}_{0}^{p}(E):=\left\{x \in P[I, E]: \varphi x \in L_{p}(I)\right\} .
$$


Further, we define the space $\mathbf{H}^{\lambda}(E), \lambda \geq 0$ by

$$
\mathbf{H}^{\lambda}(E):=\left\{x \in C\left[I, E_{\omega}\right]:\right.
$$

$\varphi x$ is Hölderain of order $\lambda$ on $I$, for every $\varphi$

$$
\left.\in E^{*}\right\}
$$

We also define $\mathbf{H}^{0}(E):=C\left[I, E_{\omega}\right]$.

In the following proposition, we summarize some important facts which are the main tool in carrying out our investigations (see $[10,12,16,17])$.

Proposition 2. Let $p, q \in[1, \infty]$ be of conjugate exponents (that is, $1 / p+1 / q=1$ with the convention that $1 / \infty=0)$. If $x: I \rightarrow E$ is weakly measurable, then

(1) if $x(\cdot) \in P[I, E]$ and $u(\cdot) \in L_{\infty}[I, \mathbb{R}]$, then the following hold $x(\cdot) u(\cdot) \in P[I, E]$,

(2) $x \in \mathscr{H}_{0}^{p}(E), p>1$ if and only if $x(\cdot) u(\cdot) \in P[I, E]$ for every $u(\cdot) \in L_{q}(I)$,

(3) if $E$ is reflexive (containing no isometric copy of $c_{0}$ ), the weakly (strongly) measurable function $x: I \rightarrow E$ is Pettis integrable on $I$ if and only if $x$ is Dunford integrable on $I$,

(4) for any $p>1$, we have $\left\{x \in \mathscr{H}^{p}(E)\right.$ : $x$ strongly measurable $\} \subseteq \mathscr{H}_{0}^{p}(E)$. If $E$ is weakly sequentially complete, this is also true for $p=1$.

We remark that there is a bounded weakly measurable function which is not Pettis integrable (see, e.g., [19]).

A fundamental property of Pettis integral is contained in the following.

Proposition 3 (see [12] Corollary 2.51). If $x \in P[I, E]$, then for any bounded subset $\Omega$ of elements of $E^{*}$, the integrals

$$
\int_{J}|\varphi(x(s))| d s, \quad \varphi \in \Omega
$$

are weakly equi-absolutely continuous.

Theorem 4 (mean value theorem for Pettis integral). If the function $x: I \rightarrow E$ is Pettis integrable on $I$, then

$$
\int_{J} x(s) d s \in|J| \overline{\operatorname{conv}}(x(J)),
$$

where $J \subset I,|J|$ is the length of $J$ and $\overline{\operatorname{conv}}(x(J))$ is the closed convex hull of $x(J)$.

\section{Fractional Integrals of Vector-Valued Functions}

In this section, we define and study the Riemann-Liouville fractional integral operators and the corresponding fractional derivatives in Banach spaces.

Devoted by the definition of the Riemann-Liouville fractional integral of real-valued function, we introduce the following.
Definition 5. Let $x: I \rightarrow E$. The Riemann-Liouville fractional Pettis integral (shortly RFPI) of $x$ of order $\alpha>0$ is defined by

$$
\mathfrak{J}^{\alpha} x(t):=\frac{1}{\Gamma(\alpha)} \int_{0}^{t}(t-s)^{\alpha-1} x(s) d s, \quad t>0 .
$$

In the preceding definition, " $\int$ " stands for the Pettis integral.

When $E=\mathbb{R}$, it is well known (see, e.g., $[20,21]$ ) that the operator $\mathfrak{J}^{\alpha}$ sends $L_{q}[0, b], b \in(0, \infty)$ continuously to $L_{p}[0, b]$ if $p \in[1, \infty]$ satisfy $q>1 /(\alpha+(1 / p))$.

This seems to be a good place to put the following.

Example 6. Let $E$ be an infinite dimensional Banach space that fails cotype (see [22] and the references therein). Define the strongly measurable function $x:[0,1] \rightarrow E$ by

$$
x(t):=\sum_{n=1}^{\infty} \sum_{k=1}^{2^{n}} c_{n} \frac{\chi_{A_{k}^{n}}(t)}{\mu\left(A_{k}^{n}\right)} e_{k}^{n}, \quad \mu: \text { Lebesgue measure }
$$

with similar notations as in ([13], Corollary 4) where we choose $c_{n}=2 K \psi\left(2^{3-n}\right), K>1, \psi(t):=t^{\epsilon}, \epsilon=0.9$ and

$$
\left\{A_{k}^{n}=\left[\frac{a_{k}}{3^{n}}, \frac{b_{k}}{3^{n}}\right]: n=0,1,2, \ldots, k=1,2,3, \ldots, 2^{n}\right\}
$$

to be the fat Cantor sets (that is, $\mu\left(A_{k}^{n}\right)=1 / 3^{n}$ holds for every $\left.k \in\left\{1,2,3, \ldots, 2^{n}\right\}\right)$.

As cited in ([13], Corollary 4), $x$ is Pettis integrable functions on $[0,1]$ whose indefinite integral is nowhere weakly differentiable on $[0,1]$. Here we will show that $x$ has RFPI of all order $\alpha \geq 3 / 4$ and

$$
\begin{aligned}
& \mathfrak{J}^{\alpha} x(t) \\
& \quad=\sum_{n=1}^{\infty} \sum_{k=1}^{2^{n}} c_{n}\left(\frac{e_{k}^{n}}{\mu\left(A_{k}^{n}\right) \Gamma(\alpha)} \int_{[0, t] \cap A_{k}^{n}}(t-s)^{\alpha-1} d s\right) \\
& \quad \in E .
\end{aligned}
$$

Arguing similarly as in ([13], page 368), we have in view of $\left\|\sum_{k=1}^{2^{n}} \varepsilon_{k}^{n} u_{k}^{n}\right\|_{\ell_{\infty}^{2^{n}}}=1$ that $\sum_{k=1}^{2^{n}}\left|\varphi\left(e_{k}^{n}\right)\right| \leq 2\|\varphi\|$ holds for every $\varphi \in E^{*}$.

Also, for any $t \in[0,1]$ and fixed $n \in \mathbb{N}$, we have for some $n_{0}<n$ that

$$
\begin{gathered}
\sum_{k=1}^{2^{n}}\left[\int_{0}^{t}(t-s)^{\alpha-1} \frac{\chi_{A_{k}^{n}}(s)}{\mu\left(A_{k}^{n}\right)} d s\right]^{2} \\
=\sum_{k=1}^{2^{n}} 3^{2 n}\left[\int_{[0, t] \cap A_{k}^{n}}(t-s)^{\alpha-1} d s\right]^{2} \\
=3^{2 n} \sum_{k=1}^{2^{n_{0}}}\left[\int_{A_{k}^{n}}(t-s)^{\alpha-1} d s\right]^{2}
\end{gathered}
$$




$$
\begin{aligned}
& +3^{2 n}\left[\int_{a_{2^{n_{0}+1}}}^{t}(t-s)^{\alpha-1} d s\right]^{2} \\
\leq & \frac{3^{2 n}}{\alpha} \sum_{k=1}^{2^{n_{0}}}\left[\left(t-\frac{a_{k}}{3^{n}}\right)^{\alpha}-\left(t-\frac{b_{k}}{3^{n}}\right)^{\alpha}\right]^{2} \\
& +\frac{3^{2 n}}{\alpha^{2}}\left(t-\frac{a_{2^{n_{0}}+1}}{3^{n}}\right)^{2 \alpha} \\
\leq & \frac{3^{2 n}}{\alpha} \sum_{k=1}^{2^{n_{0}}}\left[\left(t-\frac{a_{k}}{3^{n}}\right)-\left(t-\frac{b_{k}}{3^{n}}\right)\right]^{2 \alpha} \\
& +\frac{3^{2 n}}{\alpha^{2}}\left(\frac{b_{2^{n_{0}+1}}}{2^{n}}-\frac{a_{2^{n_{0}}+1}}{2^{n}}\right)^{2 \alpha} \leq \frac{3^{2 n} 2^{n}}{\alpha 3^{2 n \alpha}} \\
= & \frac{2^{n}}{\alpha^{2}} 3^{2 n(1-\alpha)} .
\end{aligned}
$$

Therefore, for any measurable $J \subset[0,1]$, we arrive at

$$
\begin{aligned}
\int_{[0, t] \cap} \sum_{n=1}^{\infty}\left|\sum_{k=1}^{2^{n}} \frac{c_{n}}{\Gamma(\alpha)}(t-s)^{\alpha-1} \frac{\chi_{A_{k}^{n}}(s)}{\mu\left(A_{k}^{n}\right)} \varphi\left(e_{k}^{n}\right) d s\right| \\
\leq \int_{0}^{t} \sum_{n=1}^{\infty}\left|\sum_{k=1}^{2^{n}} \frac{c_{n}}{\Gamma(\alpha)}(t-s)^{\alpha-1} \frac{\chi_{A_{k}^{n}}(s)}{\mu\left(A_{k}^{n}\right)} \varphi\left(e_{k}^{n}\right) d s\right| \\
\leq \sum_{n=1}^{\infty} \frac{c_{n}}{\Gamma(\alpha)} \int_{0}^{t}\left|\sum_{k=1}^{2^{n}}(t-s)^{\alpha-1} \frac{\chi_{A_{k}^{n}}(s)}{\mu\left(A_{k}^{n}\right)} \varphi\left(e_{k}^{n}\right) d s\right| \\
\leq \sum_{n=1}^{\infty} \frac{c_{n}}{\Gamma(\alpha)} \sum_{k=1}^{2^{n}}\left|\varphi\left(e_{k}^{n}\right)\right| \int_{0}^{t}(t-s)^{\alpha-1} \frac{\chi_{A_{k}^{n}}(s)}{\mu\left(A_{k}^{n}\right)} d s \\
\leq \sum_{n=1}^{\infty} \frac{c_{n}}{\Gamma(\alpha)}\left(\sum_{k=1}^{2^{n}}\left|\varphi\left(e_{k}^{n}\right)\right|^{2}\right)^{1 / 2} \\
.\left(\sum_{k=1}^{2^{n}}\left[\int_{0}^{t}(t-s)^{\alpha-1} \frac{\chi_{A_{k}^{n}}(s)}{\mu\left(A_{k}^{n}\right)} d s\right]^{2}\right)^{1 / 2} \leq 2\|\varphi\| \\
\quad \cdot \sum_{n=1}^{\infty} \frac{\sqrt{2^{n}} 3^{n(1-\alpha)} c_{n}}{\alpha \Gamma(\alpha)}=4 K 2^{3 \epsilon}\|\varphi\| \sum_{n=1}^{\infty} \frac{2^{n(1 / 2-\epsilon)} 3^{n(1-\alpha)}}{\Gamma(1+\alpha)} .
\end{aligned}
$$

Obviously, the latter series converges whenever $\alpha \geq 3 / 4$, $\epsilon=0.9$ which allows us to interchange the integral and summation below to see that

$$
\begin{aligned}
& \frac{1}{\Gamma(\alpha)} \int_{[0, t] \cap J} \varphi\left((t-s)^{\alpha-1} x(s)\right) \\
& =\frac{1}{\Gamma(\alpha)} \int_{[0, t] \cap J}(t-s)^{\alpha-1} \varphi(x(s)) \\
& =\int_{[0, t] \cap J} \sum_{n=1}^{\infty} \sum_{k=1}^{2^{n}} \frac{c_{n}}{\Gamma(\alpha)}(t-s)^{\alpha-1} \frac{\chi_{A_{k}^{n}}(s)}{\mu\left(A_{k}^{n}\right)} \varphi\left(e_{k}^{n}\right) d s \\
& =\sum_{n=1}^{\infty} \sum_{k=1}^{2^{n}} \int_{[0, t] \cap J} \frac{c_{n}}{\Gamma(\alpha)}(t-s)^{\alpha-1} \frac{\chi_{A_{k}^{n}}(s)}{\mu\left(A_{k}^{n}\right)} \varphi\left(e_{k}^{n}\right) d s \\
& =\varphi\left[\sum_{n=1}^{\infty} \sum_{k=1}^{2^{n}} c_{n}\left(\frac{e_{k}^{n}}{\mu\left(A_{k}^{n}\right) \Gamma(\alpha)} \int_{[0, t] \cap A_{k}^{n}}(t-s)^{\alpha-1} d s\right)\right] .
\end{aligned}
$$

It remains to prove that

$$
\sum_{n=1}^{\infty} \sum_{k=1}^{2^{n}} c_{n}\left(\frac{e_{k}^{n}}{\mu\left(A_{k}^{n}\right) \Gamma(\alpha)} \int_{[0, t] \cap A_{k}^{n}}(t-s)^{\alpha-1} d s\right) \in E .
$$

Evidently, we have

$$
\begin{aligned}
& \left\|\sum_{n=l}^{m} \sum_{k=1}^{2^{n}} e_{k}^{n} c_{n}\left(\int_{[0, t] \cap A_{k}^{n}} \frac{(t-s)^{\alpha-1}}{\mu\left(A_{k}^{n}\right) \Gamma(\alpha)} d s\right)\right\|_{E} \\
& =\left\|\sum_{n=l}^{m} \sum_{k=1}^{2^{n}} c_{n}\left(\int_{[0, t] \cap A_{k}^{n}} \frac{(t-s)^{\alpha-1}}{\mu\left(A_{k}^{n}\right) \Gamma(\alpha)} d s\right) T_{n} u_{k}^{n}\right\|_{E} \\
& \quad \leq 2 \sum_{n=l}^{m} c_{n}\left\|\sum_{k=1}^{2^{n}}\left(\int_{[0, t] \cap A_{k}^{n}} \frac{(t-s)^{\alpha-1}}{\mu\left(A_{k}^{n}\right) \Gamma(\alpha)} d s\right) u_{k}^{n}\right\|_{\ell_{\infty}^{2^{n}}} \\
& \quad=\frac{2}{\Gamma(\alpha)} \sum_{n=l}^{m} 3^{n} c_{n} \max _{1 \leq k \leq 2^{n}}\left|\int_{[0, t] \cap A_{k}^{n}}(t-s)^{\alpha-1} \chi_{A_{k}^{n}}(s) d s\right| \\
& \quad \leq \frac{2}{\Gamma(\alpha)} \sum_{n=l}^{m} 3^{n} c_{n} \max _{1 \leq k \leq 2^{n}}\left|\int_{a_{k} / 3^{n}}^{b_{k} / 3^{n}}(t-s)^{\alpha-1} d s\right| \\
& \quad \leq \frac{4 K 2^{3 \epsilon}}{\Gamma(1+\alpha)} \sum_{n=l}^{m} \frac{3^{n(1-\alpha)}}{2^{\epsilon n}},{ }^{m} \mid
\end{aligned}
$$

which approaches zero as $l, m \rightarrow \infty$ as needed for (9).

Remark 7. Observe Example 6. We remark the following:

(1) There is a reflexive Banach space for which the indefinite Pettis integral of the function $x$ defined by (7) is nowhere weakly differentiable on $[0,1]$ (see [13], the remark below Corollary 4). Meanwhile, $x$ has a RFPI of all order $\alpha \geq 3 / 4$.

(2) The function $\mathfrak{\Im}^{\alpha} x(\cdot)$ is weakly continuous on $[0,1]$ : this follows easily from the definition of the Pettis integral. In fact, we have in view of (9) that

$$
\begin{aligned}
\varphi & \left(\mathfrak{J}^{\alpha} x(t)\right)=\mathfrak{J}^{\alpha} \varphi(x(t)) \\
& =\sum_{n=1}^{\infty} \sum_{k=1}^{2^{n}} \frac{c_{n} \varphi\left(e_{k}^{n}\right)}{\mu\left(A_{k}^{n}\right) \Gamma(\alpha)} \int_{[0, t] \cap A_{k}^{n}}(t-s)^{\alpha-1} d s
\end{aligned}
$$

holds for every $\varphi \in E^{*}$. Since

$$
\begin{aligned}
& \left|\sum_{k=1}^{2^{n}} \frac{c_{n} \varphi\left(e_{k}^{n}\right)}{\mu\left(A_{k}^{n}\right) \Gamma(\alpha)} \int_{[0, t] \cap A_{k}^{n}}(t-s)^{\alpha-1} d s\right| \\
& \quad \leq c_{n}\left(\sum_{k=1}^{2^{n}}\left|\varphi\left(e_{k}^{n}\right)\right|^{2}\right)^{1 / 2} \\
& \quad\left(\sum_{k=1}^{2^{n}}\left[\int_{[0, t] \cap A_{k}^{n}} \frac{(t-s)^{\alpha-1}}{\mu\left(A_{k}^{n}\right) \Gamma(\alpha)} d s\right]^{2}\right)^{1 / 2} \\
& \quad \leq 4 K 2^{3 \epsilon}\|\varphi\| \frac{2^{n(1 / 2-\epsilon)} 3^{n(1-\alpha)}}{\Gamma(1+\alpha)}
\end{aligned}
$$


then the infinite series of continuous functions in the left hand side of (15) converges uniformly in $[0,1]$. Hence, the function $\varphi\left(\mathfrak{I}^{\alpha} x(\cdot)\right)$ is continuous on $[0,1]$ (this yields the weak continuity of $\mathfrak{J}^{\alpha} x(\cdot)$ on $[0,1]$ ).

In the following lemma, we gather together some simple particular sufficient conditions that ensure the existence of the Riemann-Liouville fractional integral of the functions from $\mathscr{H}^{p}(E)$.

Lemma 8. Let $x: I \rightarrow E$ be weakly measurable function. The RFPI of the function $x$ of order $\alpha>0$ makes sense a.e. on I if at least one of the following cases holds:

(a) $x \in \mathscr{H}_{0}^{p}(E), p \in[1, \infty]$ and $\alpha \geq 1$.

(b) $x \in \mathscr{H}_{0}^{p}(E), p>\max \{1,1 / \alpha\}$.

(c) $x$ is strongly measurable which lies in $\mathscr{H}^{p}(E)$, where $p \in[1, \infty]$ provided that $E$ is weakly complete or contains no copy of $c_{0}$.

If $E$ is reflexive, this is also true for any $x \in \mathscr{H}^{p}(E), p \in[1, \infty]$ and $\alpha>0$.

In all cases, $\varphi\left(\mathfrak{\Im}^{\alpha} x\right)=\mathfrak{\Im}^{\alpha} \varphi x$ holds for every $\varphi \in E^{*}$.

In the assertions ((a) and (b)), we find sufficient conditions needed for the existence of $\mathfrak{J}^{\alpha} x$ in the situation in which no restriction is placed on $E$. In the third assertion, the properties of $E$ allow us to characterize a function $x \in \mathscr{H}^{p}(E)$ for which $\mathfrak{\Im}^{\alpha} x$ exists.

Proof. Firstly, assertion (a) is direct consequence of Proposition 2 (part (1)) since $s \rightarrow(t-s)^{\alpha-1} \in L_{\infty}[0, t]$ holds for almost every $t \in I$ whenever $\alpha \geq 1$.

Secondly, to prove assertion (b), let $p>\max \{1,1 / \alpha\}$ and $q$ be the conjugate exponents to $p$. Since $q(\alpha-1)+1>0$, we have that $s \rightarrow(t-s)^{\alpha-1} \in L_{q}[0, t]$ holds for every $t \in I$. Thus, the assertion (b) is now an easy consequence of Proposition 2 (part (2)).

Thirdly, to prove (c) we let $\alpha>0, p \in[1, \infty]$ and $x \in \mathscr{H}^{p}(E)$ be strongly measurable. Since the strong measurability is preserved under multiplication operation on functions, the product $(t-\cdot)^{\alpha-1} x(\cdot):[0, t] \rightarrow E$ is strongly measurable on $[0, t]$ for almost every $t \in I$. In view of Young's inequality, for every $\varphi \in E^{*}$, the real-valued function,

$$
s \longmapsto \varphi\left((t-s)^{\alpha-1} x(s)\right)=(t-s)^{\alpha-1} \varphi x(s),
$$

is Lebesgue integrable on $[0, t]$, for almost every $t \in I$. So the assertion (c) follows immediately from Proposition 2 (parts $(3,4))$.

Similarly, when $E$ is reflexive, the result follows from part (3) of Proposition 2.

However, in all cases, the function $s \mapsto(t-s)^{\alpha-1} x(s)$ is Pettis integrable on $[0, t]$ for almost every $t \in I$. That is, for almost every $t \in I$, there exists an element in $E$ denoted by $\mathfrak{\Im}^{\alpha} x(t)$ such that

$$
\varphi\left(\mathfrak{J}^{\alpha} x(t)\right)=\int_{0}^{t} \varphi\left(\frac{(t-s)^{\alpha-1}}{\Gamma(\alpha)} x(s)\right) d s
$$

$$
=\int_{0}^{t} \frac{(t-s)^{\alpha-1}}{\Gamma(\alpha)} \varphi x(s) d s=\mathfrak{J}^{\alpha} \varphi x(t)
$$

holds for every $\varphi \in E^{*}$. This completes the proof.

Remark 9. If $x \in \mathscr{H}_{0}^{p}(E)$ such that $\mathfrak{\Im}^{\alpha} x(t)$ does not exist for some $t \in I$, then it does not exist even when we "enlarge" the space $E$ into $F$. To see this, let $x: I \rightarrow F$ such that $x(I) \subset E$. If $\mathfrak{\Im}^{\alpha} x(t)$ exists for some $t \in I$, then $(t-\cdot)^{\alpha-1} x(\cdot) \in P([0, t], F)$. Since $x$ assumes only values in $E$, it follows by the mean value theorem for Pettis integral (Theorem 4) that the RFPI of $x$ should lie in $E$.

Before we come to a deep study of the mathematical properties of the RFPI operator, let us take a look at the following miscellaneous examples.

Example 10. Let $\alpha>0$. Define the function $x:[0,1] \rightarrow$ $L_{\infty}[0,1]$ by $x(t)=\chi_{[0, t]}$.

This function is weakly measurable, Pettis integrable on $[0,1]$, and $\varphi x$ is a function of bounded variation (see, e.g., [18]). That is, $x \in \mathscr{H}_{0}^{\infty}\left(L_{\infty}\right)$. Hence, in view of Lemma 8 with $p=\infty$, the RFPI of $x$ exists on $[0,1]$. Further, calculations (cf. [4]) show that

$$
\mathfrak{\Im}^{\alpha} x(t)(\cdot)=\frac{(t-\cdot)^{\alpha}}{\Gamma(1+\alpha)} \chi_{[0, t]}(\cdot) \in L_{\infty}[0,1]
$$

Example 11. Let $\alpha>0$. Define the function $x$ from the interval $[0,1]$ into the Hilbert space $\ell_{2}$ as

$$
x: t \longmapsto\left\{\frac{1}{(1+t)}, \frac{1}{(2+t)}, \frac{1}{(3+t)}, \ldots\right\}, \quad t \in[0,1] .
$$

We note that

$$
\|x(t)\|_{\ell_{2}}^{2}=\sum_{n}\left(\frac{1}{n+t}\right)^{2} \leq \sum_{n} \frac{1}{n^{2}}<\infty, \quad t \in[0,1] .
$$

Thus, the function $x$ is well defined. We claim that $x$ is Dunford integrable on $[0,1]$. Once our claim is established, Lemma 8 guarantees the existence of $\mathfrak{\Im}^{\alpha} x$ on $[0,1]$. It remains to prove this claim and to calculate $\mathfrak{I}^{\alpha} x$.

To see this, let $\varphi \in\left(\ell_{2}\right)^{*}=\ell_{2}$. According to the Riesz representation theorem on Hilbert spaces there exists a uniquely determined $\lambda:=\left\{\lambda_{n}\right\} \in \ell_{2}$ such that $\varphi x(t)=$ $\sum_{n}\left(\lambda_{n} /(n+t)\right)$. A standard arguments using Beppo Levi's Theorem yields

$$
\begin{aligned}
\int_{0}^{1}|\varphi x(t)| d t & \leq \sum_{n} \int_{0}^{1} \frac{\left|\lambda_{n}\right|}{(n+t)} d t=\sum_{n}\left|\lambda_{n}\right| \ln \left(1+\frac{1}{n}\right) \\
& \leq \sum_{n} \frac{\left|\lambda_{n}\right|}{n} \leq \sum_{n}\left|\lambda_{n}\right|<\infty .
\end{aligned}
$$

So $x$ is Dunford integrable on $[0,1]$ and hence Pettis integrable on $[0,1]$ since $\ell_{2}$ is reflexive. Consequently, in view of 
Journal of Function Spaces

5

Lemma $8, \mathfrak{\Im}^{\alpha} x$ exists on $[0,1]$. To calculate the RFPI of $x$ fix $t \in[0,1]$ and let $\varphi \in\left(\ell_{2}\right)^{*}$. We have

$$
\begin{aligned}
& \int_{0}^{t} \varphi\left(\frac{(t-s)^{\alpha-1}}{\Gamma(\alpha)} x(s)\right) d s=\int_{0}^{t} \frac{(t-s)^{\alpha-1}}{\Gamma(\alpha)} \varphi x(s) d s \\
& \quad=\mathfrak{J}^{\alpha}\left(\sum_{n=1}^{\infty} \frac{\lambda_{n}}{(n+t)}\right) .
\end{aligned}
$$

Since the series $\sum_{n}\left(\lambda_{n} /(n+t)\right)$ is uniformly convergent on $[0,1]$, it follows in view of the generalized linearity of the fractional integrals [23, Lemma 5], that (cf. [21, Table 9.1])

$$
\begin{aligned}
\int_{0}^{t} \varphi\left(\frac{(t-s)^{\alpha-1}}{\Gamma(\alpha)} x(s)\right) d s \\
\quad=\sum_{n=1}^{\infty} \frac{\lambda_{n} t^{\alpha}}{n \Gamma(1+\alpha)}{ }_{2} \mathrm{~F}_{1}\left(1,1, \alpha+1, \frac{-t}{n}\right)=\varphi(g(t)),
\end{aligned}
$$

where ${ }_{2} \mathrm{~F}_{1}$ is the Gauss hypergeometric function evaluated at $(1,1, \alpha+1,-t / n)$ and

$$
g(t):=\left\{\frac{t^{\alpha}}{n \Gamma(1+\alpha)}{ }_{2} \mathrm{~F}_{1}\left(1,1, \alpha+1, \frac{-t}{n}\right)\right\} .
$$

Since

$$
\begin{aligned}
&\|g(t)\|_{\ell_{2}}^{2}=\sum_{n}\left(\frac{t^{\alpha}}{\Gamma(1+\alpha)}{ }_{2} \mathrm{~F}_{1}\left(1,1, \alpha+1, \frac{-t}{n}\right)\right)^{2} \\
& \leq \frac{{ }_{2} \mathrm{~F}_{1}^{2}(1,1, \alpha+1,-1)}{\Gamma^{2}(1+\alpha)} \sum_{n} \frac{1}{n^{2}}<\infty, \\
& \quad t \in[0,1],
\end{aligned}
$$

we see that $g \in \ell_{2}$. Thus,

$$
\begin{aligned}
\mathfrak{J}^{\alpha} x(t)=\left\{\frac{t^{\alpha}}{n \Gamma(1+\alpha)}{ }_{2} \mathrm{~F}_{1}\left(1,1, \alpha+1, \frac{-t}{n}\right)\right\} & , \\
& t \in[0,1] .
\end{aligned}
$$

Example 12. Let $\alpha \in[1 / 2,1)$. Define the countable-valued function $x:[0,1] \rightarrow c_{0}$ by

$$
x(t):=\left\{x_{n}(t)\right\}, \quad x_{n}:=n \chi_{(1 /(n+1), 1 / n]} .
$$

This function is strongly measurable, Pettis integrable functron on $[0,1]$ (see, e.g., $[10,16])$. We claim that $x \in \mathscr{H}_{0}^{P}\left(c_{0}\right)$ with $p \in(1 / \alpha, 2]$. Once our claim is established, Lemma 8 guarantees the existence of $\mathfrak{J}^{\alpha} x, \alpha \in[1 / 2,1)$ on $[0,1]$. It remains to prove this claim by showing firstly that $\mathscr{H}^{P}\left(c_{0}\right)$ and to calculate $\mathfrak{J}^{\alpha} x$. To do this, let $\varphi \in c_{0}^{*}$. Then there corresponds to $\varphi$ a unique $\left\{\lambda_{n}\right\} \in \ell_{1}$ such that $\varphi x=\sum_{n} \lambda_{n} x_{n}$. By noting that

$$
\begin{aligned}
\left\|\lambda_{n} x_{n}\right\|_{p} & =\left(\int_{0}^{1}\left|\lambda_{n} x_{n}(t)\right|^{p} d t\right)^{1 / p} \\
& =\left|\lambda_{n}\right|\left(\int_{1 /(n+1)}^{1 / n} n^{p} d t\right)^{1 / p}=\frac{\left|\lambda_{n}\right| n^{1 / q}}{(n+1)^{1 / p}} \\
& \leq \frac{\left|\lambda_{n}\right| n^{1 / q}}{n^{1 / p}} \leq \frac{\left|\lambda_{n}\right|}{n^{2 / p-1}} \leq\left|\lambda_{n}\right|
\end{aligned}
$$

holds for any $p \in(1 / \alpha, 2]$, a standard argument using Levi's Theorem (or Lebesgue dominated convergence theorem) and Minkowski's inequality yields

$$
\begin{aligned}
& {\left[\int_{0}^{1}|\varphi x(t)|^{p} d t\right]^{1 / p} \leq\left[\int_{0}^{1}\left(\sum_{n=1}^{\infty}\left|\lambda_{n}\right| x_{n}(t)\right)^{p} d t\right]^{1 / p}} \\
& \quad=\left[\lim _{k \rightarrow \infty} \int_{0}^{1}\left(\sum_{n=1}^{k}\left|\lambda_{n}\right| x_{n}(t)\right)^{p} d t\right]^{1 / p} \\
& \quad \leq\left[\lim _{k \rightarrow \infty}\left(\sum_{n=1}^{k}\left\|\lambda_{n} x_{n}\right\|_{p}\right)^{p}\right]^{1 / p} \leq \sum_{n=1}^{\infty}\left\|\lambda_{n} x_{n}\right\|_{p} \\
& \quad \leq \sum_{n=1}^{\infty}\left|\lambda_{n}\right|<\infty
\end{aligned}
$$

Thus, $\varphi x(\cdot) \in L_{p}[0,1], p \in(1 / \alpha, 2]$ holds for every $\varphi \in c_{0}^{*}$ (that is, $x \in \mathscr{H}^{P}\left(c_{0}\right)$ ). Since $p>1 / \alpha>1$, it follows by Proposition 2, in view of the strong measurability of $x$, that $x \in \mathscr{H}_{0}^{P}\left(c_{0}\right)$. Owing to Lemma 8 , we infer that the RFPI of $x$ of any order $\alpha \in[1 / 2,1)$ exists on the interval $[0,1]$. To compute this integral, fix $t \in I_{n_{0}}:=\left[1 /\left(n_{0}+1\right), 1 / n_{0}\right]$ for some $n_{0} \in \mathbb{N}$, the set of natural numbers, and let $\varphi \in c_{0}^{*}$. Then

$$
\begin{aligned}
& \sum_{n} \int_{0}^{t}\left|(t-s)^{\alpha-1} \lambda_{n}\right| x_{n}(s) d s \\
& \leq|| \lambda_{n_{0}} \mid \int_{1 /\left(n_{0}+1\right)}^{t}(t-s)^{\alpha-1} n_{0} d s \\
& \quad+\sum_{n>n_{0}}\left|\lambda_{n}\right| \int_{1 /(n+1)}^{1 / n}(t-s)^{\alpha-1} n d s \\
&=\frac{n_{0}\left|\lambda_{n_{0}}\right|}{\alpha}\left(t-\frac{1}{n_{0}+1}\right)^{\alpha} \\
& \quad+\sum_{n>n_{0}} \frac{n\left|\lambda_{n}\right|}{\alpha}\left[\left(t-\frac{1}{n+1}\right)^{\alpha}-\left(t-\frac{1}{n}\right)^{\alpha}\right] \\
& \leq \frac{n_{0}\left|\lambda_{n_{0}}\right|}{\alpha}\left(t-\frac{1}{n_{0}+1}\right)^{\alpha} \\
& \leq \frac{n_{0}\left|\lambda_{n_{0}}\right|}{\alpha}\left(t-\frac{1}{n_{0}+1}\right)^{\alpha}+\sum_{n>n_{0}} \frac{\left|\lambda_{n}\right|}{\alpha}<\infty . \\
& \leq \frac{\sum_{n>n_{0}} \frac{n\left|\lambda_{n}\right|}{\alpha}\left[\left(t-\frac{1}{n+1}\right)-\left(t-\frac{1}{n}\right)\right]^{\alpha}}{\alpha}\left(t-\frac{1}{n_{0}+1}\right)^{\alpha}+\lambda_{n>n_{0}} \frac{\left|\lambda_{n}\right|}{\alpha} \frac{1}{n^{2 \alpha-1}} \\
& \leq\left(t-\frac{1}{n_{0}+1}\right)^{\alpha}+\sum_{n>n_{0}} \frac{n\left|\lambda_{n}\right|}{\alpha} \frac{1}{n^{\alpha}(n+1)^{\alpha}}
\end{aligned}
$$


Therefore, by Beppo Levi Theorem it follows that

$$
\begin{aligned}
\int_{0}^{t} \varphi & \left(\frac{(t-s)^{\alpha-1}}{\Gamma(\alpha)} x(s)\right) d s=\int_{0}^{t} \frac{(t-s)^{\alpha-1}}{\Gamma(\alpha)} \varphi(x(s)) d s \\
= & \int_{0}^{t} \frac{(t-s)^{\alpha-1}}{\Gamma(\alpha)} \sum_{n} \lambda_{n} n \chi_{(1 /(n+1), 1 / n]}(s) d s \\
= & \sum_{n} \int_{0}^{t} \frac{(t-s)^{\alpha-1}}{\Gamma(\alpha)} \lambda_{n} n \chi_{(1 /(n+1), 1 / n]}(s) d s \\
= & \frac{n_{0} \lambda_{n_{0}}}{\Gamma(1+\alpha)}\left(t-\frac{1}{n_{0}+1}\right)^{\alpha} \\
& +\sum_{n>n_{0}} \frac{n \lambda_{n}}{\Gamma(1+\alpha)}\left[\left(t-\frac{1}{n+1}\right)^{\alpha}-\left(t-\frac{1}{n}\right)^{\alpha}\right] .
\end{aligned}
$$

Consequently, we conclude that

$$
\begin{aligned}
& \mathfrak{\Im}^{\alpha} x(t)=\{\underbrace{0, \ldots, 0}_{n_{0}-1}, \frac{n_{0}}{\Gamma(1+\alpha)}\left(t-\frac{1}{n_{0}+1}\right)^{\alpha}, \\
& \left.\quad \frac{\left(n_{0}+1\right)}{\Gamma(1+\alpha)}\left[\left(t-\frac{1}{n_{0}+2}\right)^{\alpha}-\left(t-\frac{1}{n_{0}+1}\right)^{\alpha}\right], \ldots\right\},
\end{aligned}
$$

where the nonzero coordinate started from the $n_{0}$ th place. It can be easily seen that $\mathfrak{J}^{\alpha} x(t) \in c_{0}$ for any $t \in[0,1]$. Evidently, we have that

$$
\begin{aligned}
& \left|n\left[\left(t-\frac{1}{n+1}\right)^{\alpha}-\left(t-\frac{1}{n}\right)^{\alpha}\right]\right| \\
& \quad \leq n\left[\left(t-\frac{1}{n+1}\right)-\left(t-\frac{1}{n}\right)\right]^{\alpha} \leq n\left[\frac{1}{n(n+1)}\right]^{\alpha} \\
& \quad \leq \frac{1}{n^{2 \alpha-1}} \longrightarrow 0 \text { as } n \longrightarrow \infty, \\
& \frac{n_{0}}{\Gamma(1+\alpha)}\left(t-\frac{1}{n_{0}+1}\right)^{\alpha} \\
& \quad \leq \frac{n_{0}}{\Gamma(1+\alpha)}\left(\frac{1}{n_{0}}-\frac{1}{n_{0}+1}\right)^{\alpha} \leq \frac{1}{n_{0}^{2 \alpha-1} \Gamma(1+\alpha)} \\
& \quad \longrightarrow 0 \text { as } n_{0} \longrightarrow \infty
\end{aligned}
$$

hold for any $t \in[0,1]$. This yields $\mathfrak{\Im}_{1}^{\alpha} x(t) \in \mathcal{c}_{0}$ for any $t \in[0,1]$ (this is precisely what we would expect from Definition 5).

Look at part (c) of Lemma 8, with $\alpha \in(0,1)$ and $E=c_{0}$. Below we give an example showing that the strongly measurability hypothesis imposed on a function $x \in \mathscr{H}^{1}(E) /$ $\mathscr{H}_{0}^{1}(E)$ is not sufficient for the existence of $\mathfrak{\Im}^{\alpha} x$ even when $x$ is Denjoy-Pettis integrable (cf. [16]).

Example 13. Let $\alpha \in(0,1)$. Define the strongly measurable function $x:[0,1] \rightarrow c_{0}$ by

$$
\begin{aligned}
x(t):=\left\{x_{n}(t)\right\}, & \\
& x_{n}(t):=n(n+1)\left[\chi_{I_{n}}(t)-\chi_{J_{n}}(t)\right],
\end{aligned}
$$

where

$$
\begin{aligned}
& I_{n}:=\left(\frac{1}{n+1}, \frac{n+1 / 2}{n(n+1)}\right), \\
& J_{n}:=\left(\frac{n+1 / 2}{n(n+1)}, \frac{1}{n}\right) .
\end{aligned}
$$

It is immediate that (cf. [16]) $x$ is a well-defined, DenjoyPettis (but it is not Pettis) integrable on $[0,1]$.

In what follows, we will show that the RFPI of $x$ does not exist on a subinterval of positive measure on $[0,1]$.

To see this, we make use of Proposition 3 as follows: define for each interval $\mathscr{I}_{n}=[1 /(n+1), 1 / n]$ the functionals $\varphi_{n} \in c_{0}^{*}$ (required by Proposition 3 ) to be the corresponding to the element $\left\{\lambda_{n}\right\}:=(0,0, \ldots, 0,1,0,0, \ldots) \in \ell_{1}$ where the nonzero coordinate is in the $n$th place. Then $\varphi_{n}(x(t))=$ $x_{n}(t)$ and thus $\left|\varphi_{n}(x(s))\right|=n(n+1)\left[\chi_{I_{n}}(t)-\chi_{J_{n}}(t)\right]$. Clearly, the family $\left\{\varphi_{n}\right\}$ runs through the unit ball of the dual of $c_{0}$. Evidently, the isometric isomorphism between $c_{0}^{*}$ and $\ell_{1}$ yields $\left\|\varphi_{n}\right\|_{c_{0}^{*}}=\left\|\left\{\lambda_{n}\right\}\right\|_{\ell_{1}}=1$.

Now take $t \in[1 / 2,1]$ and define the continuous function $f:[1 / 2,1] \rightarrow \mathbb{R}^{+}$by

$$
\begin{aligned}
f(t):=\left(t-\frac{1}{n+1}\right)^{\alpha}-\left(t-\frac{1}{n}\right)^{\alpha}, & \\
& n \geq 2, t \in\left[\frac{1}{2}, 1\right] .
\end{aligned}
$$

It can be easily seen that, in view of $0<\alpha<1, f^{\prime}$ is negative on $(0,1)$. By standard results from (classical) calculus, it follows that $f$ is strictly decreasing on $[0,1]$, in particular $f(1)<f(t)$ for all $t \in[0,1)$. Thus, for any $n \geq 2$ and any $\varphi \in\left\{\varphi_{n}\right\}$ we have

$$
\begin{aligned}
\int_{\mathscr{F}_{n}}\left|\varphi\left(\frac{(t-s)^{\alpha-1}}{\Gamma(\alpha)} x(s)\right)\right| d s \\
=\int_{\mathscr{F}_{n}} \frac{(t-s)^{\alpha-1}}{\Gamma(\alpha)}|\varphi(x(s))| d s \\
=\int_{1 /(n+1)}^{1 / n} \frac{(t-s)^{\alpha-1}}{\Gamma(\alpha)} n(n+1) d s \\
=\frac{n(n+1)}{\Gamma(1+\alpha)}\left[\left(t-\frac{1}{n+1}\right)^{\alpha}-\left(t-\frac{1}{n}\right)^{\alpha}\right] \\
\geq \frac{n^{2}}{\Gamma(1+\alpha)}\left[\left(1-\frac{1}{n+1}\right)^{\alpha}-\left(1-\frac{1}{n}\right)^{\alpha}\right] .
\end{aligned}
$$

An explicit calculation using L'Hospital's rule two times reveals

$$
\begin{aligned}
\lim _{n \rightarrow \infty} \frac{(1-1 /(n+1))^{\alpha}-(1-1 / n)^{\alpha}}{n^{-2}}=\frac{-\alpha}{2} \\
\cdot \lim _{n \rightarrow \infty} n^{3}\left[\left(\frac{n}{n+1}\right)^{\alpha-1} \frac{1}{(n+1)^{2}}-\left(1-\frac{1}{n}\right)^{\alpha-1} \frac{1}{n^{2}}\right] \\
=\frac{-\alpha}{2} \lim _{n \rightarrow \infty} n\left[\left(\frac{n}{n+1}\right)^{\alpha-1} \frac{n^{2}}{(n+1)^{2}}-\left(1-\frac{1}{n}\right)^{\alpha-1}\right]
\end{aligned}
$$




$$
\begin{aligned}
& =\frac{-\alpha}{2} \lim _{n \rightarrow \infty} n\left[\left(\frac{n}{n+1}\right)^{\alpha+1}-\left(\frac{1}{n}\right)^{\alpha-1}\right]=\frac{-\alpha}{2} \\
& \cdot \lim _{n \rightarrow \infty} n^{2}\left[(\alpha+1)\left(\frac{n}{n+1}\right)^{\alpha} \frac{1}{(n+1)^{2}}\right. \\
& \left.-(\alpha-1)\left(1-\frac{1}{n}\right)^{\alpha-2} \frac{1}{n^{2}}\right]=\frac{-\alpha}{2} \\
& \cdot \lim _{n \rightarrow \infty}\left[(\alpha+1)\left(\frac{n}{n+1}\right)^{\alpha+2}-(\alpha-1)\left(1-\frac{1}{n}\right)^{\alpha-2}\right] \\
& =\alpha,
\end{aligned}
$$

from which it follows that

$$
\int_{\mathscr{I}_{n}}\left|\varphi\left(\frac{(t-s)^{\alpha-1}}{\Gamma(\alpha)} x(s)\right)\right| d s \nrightarrow 0 \quad \text { as } n \longrightarrow \infty \text {. }
$$

Therefore, in view of Proposition 3, $s \mapsto(t-s)^{\alpha-1} x(s) \notin$ $P\left([0,1], c_{0}\right)$. Hence, $\mathfrak{\Im}^{\alpha} x$ does not exist, which is what we wished to show.

The following theorem provides a useful characterization of the space $\mathscr{H}^{p}(E)$, for which the statements reveal how much the fractional integral $\mathfrak{J}^{\alpha}$ is better than the function $x \in \mathscr{H}^{p}(E)$. Indeed, based on Lemma 8 using an inequality of Young, we can easily prove the following.

Lemma 14. For any $\alpha>0$, the following holds.

(a) If $E$ is reflexive, then for every $p \in[1, \infty]$, the operator $\mathfrak{J}^{\alpha}$ maps $\mathscr{H}^{p}(E)$ into $\mathscr{H}_{0}^{p}(E)$. In particular, if $0<\alpha<$ 1 , then $\mathfrak{\Im}^{\alpha}: \mathscr{H}^{1}(E) \rightarrow \mathscr{H}_{0}^{1 /((1-\alpha)-\epsilon)}(E)$ however small $\epsilon>0$ is.

(b) If E contains no copy of $c_{0}$, then for every $p \in[1, \infty]$, the operator $\mathfrak{J}^{\alpha}$ maps $\left\{x \in \mathscr{H}_{0}^{p}(E): x\right.$ strongly measurable $\}$ into $\mathscr{H}_{0}^{p}(E)$. In particular, if $\alpha \geq 1$, the operator $\mathfrak{\Im}^{\alpha}$ maps $\mathscr{H}_{0}^{p}(E)$ into itself.

(c) If $p>\max \{1,1 / \alpha\}$, the operator $\mathfrak{\Im}^{\alpha}$ sends $\mathscr{H}_{0}^{p}(E)$ to $C\left[I, E_{\omega}\right]$ (if we define $\mathfrak{J}^{\alpha} x(0):=0$ ). In particular, if $\alpha \in(0,1), \mathfrak{J}^{\alpha}: \mathscr{H}_{0}^{p}(E) \rightarrow \mathbf{H}^{\alpha-1 / p}(E)$.

Proof. At the beginning, let $\alpha>0$ and $x \in \mathscr{H}^{p}(E)$ with $p \in[1, \infty]$ and define the real-valued function $g: I \rightarrow \mathbb{R}$ by $g(t):=t^{\alpha-1} / \Gamma(\alpha)$. If $E$ is reflexive, it follows, in view of Lemma 8, that $\mathfrak{J}^{\alpha} x$ exists a.e. in $I$ and $\varphi\left(\mathfrak{J}^{\alpha} x\right)=\mathfrak{I}^{\alpha} \varphi x$ for every $\varphi \in E^{*}$. Young's inequality guarantees that the convolution product $g * \varphi x(\cdot)$ lies in $L_{p}[I]$. Consequently $\varphi\left(\mathfrak{J}^{\alpha} x\right)=\mathfrak{J}^{\alpha} \varphi x=g * \varphi x \in L_{p}[I]$ for every $\varphi \in E^{*}$. The reflexivity of $E$ together with Proposition 2 yields $\mathfrak{I}^{\alpha} x \in$ $\mathscr{H}_{0}^{p}(E)$.

In particular, if $0<\alpha<1$, it can be easily seen that $\int_{I}(g(t))^{q} d t<\infty$ for every $q \in[1,1 /(1-\alpha))$. That is, $g \in$ $L_{1 /(1-\alpha)-\epsilon}[I], \epsilon>0$. By Young's inequality, it follows that $\varphi\left(\mathfrak{\Im}^{\alpha} x\right)=g * \varphi x \in L_{1 /(1-\alpha)-\epsilon}[I]$ for every $\varphi \in E^{*}$ however small $\epsilon>0$ is. Now, the assertion (a) follows because of the reflexivity of $E$.
Next, in order to prove the assertion (b) let $x \in \mathscr{H}_{0}^{p}(E)$ and note, in view of Lemma 8 , that $\mathfrak{\Im}^{\alpha} x$ exists on $I$. Define $y: I \rightarrow E$ by $y(t):=\mathfrak{\Im}^{\alpha} x(t), x \in \mathscr{H}_{0}^{p}(E)$. Since $\varphi y(\cdot) \in L_{p}[I]$ for every $\varphi \in E^{*}$, it follows that $y \in \mathscr{H}^{p}(E)$. Moreover, for any $a, b \in I(a<b)$, we have

$$
\begin{aligned}
& \int_{a}^{b} \varphi y(s) d t=\int_{a}^{b} \Im \varphi x(t) d t=\frac{1}{\Gamma(\alpha)} \\
& \cdot \int_{a}^{b} \int_{0}^{t}(t-s)^{\alpha-1} \varphi x(s) d s d t \\
& =\frac{1}{\Gamma(\alpha)}\left[\int_{a}^{b} \int_{s}^{b}(t-s)^{\alpha-1} \varphi x(s) d t d s\right. \\
& \left.+\int_{0}^{a} \int_{a}^{b}(t-s)^{\alpha-1} \varphi x(s) d t d s\right] \\
& =\frac{1}{\Gamma(\alpha)}\left[\int_{a}^{b}(b-s)^{\alpha} \varphi x(s) d s\right. \\
& \left.+\int_{0}^{a}\left[(b-s)^{\alpha}-(a-s)^{\alpha}\right] \varphi x(s) d s\right] \\
& =\frac{1}{\Gamma(\alpha)}\left[\int_{0}^{a}(b-s)^{\alpha} \varphi x(s) d s\right. \\
& \left.+\int_{a}^{b}(b-s)^{\alpha} \varphi x(s) d s-\int_{0}^{a}(a-s)^{\alpha} \varphi x(s) d s\right] \\
& =\int_{0}^{b} \frac{(b-s)^{\alpha}}{\Gamma(1+\alpha)} \varphi x(s) d s-\int_{0}^{a} \frac{(a-s)^{\alpha}}{\Gamma(1+\alpha)} \varphi x(s) d s \\
& \quad \varphi(x, b],
\end{aligned}
$$

where

$$
\begin{aligned}
x_{[a, b]}:= & (P) \int_{0}^{b} \frac{(b-s)^{\alpha}}{\Gamma(1+\alpha)} x(s) d s \\
& -(P) \int_{0}^{a} \frac{(a-s)^{\alpha}}{\Gamma(1+\alpha)} x(s) d s .
\end{aligned}
$$

Since $x \in P[I, E]$, owing to Proposition 2 (part (1)), it follows that $(b-\cdot)^{\alpha} x(s)$ and $(a-\cdot)^{\alpha} x(s)$ are Pettis integrable on $I$ and so $x_{[a, b]} \in E$.

A combination of these results yields $\varphi y \in L_{p}(I)$ for every $\varphi \in E^{*}$ and there exists an element $x_{[a, b]} \in E$ such that $\phi x_{[a, b]}=\int_{a}^{b} \varphi y(t) d t$ for every $a, b \in I$. Since $E$ contains no copy of $c_{0}$, it follows that (cf. [17, Theorem 23.]) $y \in P[I, E]$. Consequently $y(\cdot) \in \mathscr{H}_{0}^{p}(E)$. This is the claim (b).

To prove the assertion (c), let $p>\max \{1,1 / \alpha\}$ and $x \in$ $\mathscr{H}_{0}^{p}(E)$. By Lemma 8 , we deduce that $\mathfrak{\Im}^{\alpha} x$ exists a.e. on $I$. Now, let $q \in[1, \infty]$ be such that $1 / p+1 / q=1$. Since $q(\alpha-1)>-1, g:=(\cdot)^{\alpha-1} / \Gamma(\alpha) \in L_{q}[I]$. Therefore, as a direct consequence of Young' inequality it follows that

$$
\varphi\left(\mathfrak{J}^{\alpha} x(\cdot)\right)=\mathfrak{J}^{\alpha} \varphi x(\cdot)=g * \varphi x(\cdot) \in C[I, \mathbb{R}]
$$

holds for every $\varphi \in E^{*}$. Now, we claim that $\mathfrak{\Im}^{\alpha} x(t) \rightarrow 0$ in $E$ as $t \rightarrow 0$. Once our claim is established, the definition 
$\mathfrak{J}^{\alpha} x(0):=0$ guarantees that $\mathfrak{\Im}^{\alpha} x(\cdot) \in C\left[I, E_{\omega}\right]$, which is what we wished to show. It remains to prove our claim: without loss of generality, assume that $\mathfrak{J}^{\alpha} x(t) \neq 0$. Then there exists (as a consequence of the Hahn-Banach theorem) $\varphi \in E^{*}$ with $\|\varphi\|=1$ such that $\left\|\mathfrak{J}^{\alpha} x(t)\right\|=\varphi\left(\mathfrak{J}^{\alpha} x(t)\right)=\mathfrak{J}^{\alpha} \varphi x(t)$. By Hölder's inequality we obtain

$$
\begin{aligned}
& \left|\varphi\left(\mathfrak{\Im}^{\alpha} x(t)\right)\right| \\
& \leq \frac{1}{\Gamma(\alpha)}\left(\int_{0}^{t}(t-s)^{q(\alpha-1)} d s\right)^{1 / q}\left(\int_{0}^{t}|\varphi x(s)|^{p} d s\right)^{1 / p} \\
& \leq \frac{1}{\Gamma(\alpha)}\left(\frac{t^{(\alpha-1) q+1}}{(\alpha-1) q+1}\right)^{1 / q}\left(\int_{I}|\varphi x(s)|^{p} d s\right)^{1 / p} \\
& =\frac{t^{\alpha-1+1 / q}}{\Gamma(\alpha)[q(\alpha-1)+1]^{1 / q}}\|\varphi x\|_{L_{p}} \\
& =\frac{t^{\alpha-1 / p}\|\varphi x\|_{L_{p}}}{\Gamma(\alpha)[q(\alpha-1)+1]^{1 / q}} .
\end{aligned}
$$

This is equivalent with the following estimate:

$$
\left\|\boldsymbol{J}^{\alpha} x(t)\right\| \leq \frac{t^{\alpha-1 / p}\|\varphi x\|_{L_{p}}}{\Gamma(\alpha)[q(\alpha-1)+1]^{1 / q}} .
$$

Owing to $\alpha>1 / p$ we get $\mathfrak{J}^{\alpha} x(t) \rightarrow 0$ in $E$ as $t \rightarrow 0$ and consequently, in view of $\mathfrak{J}^{\alpha} x(0):=0, \mathfrak{J}^{\alpha} x(\cdot) \in C\left[I, E_{\omega}\right]$. This proves the first part of the assertion (c).

Finally, let $\alpha \in(0,1)$ and $t, \tau \in I$. Without loss of generality, assume $\tau<t$. Then for any $\varphi \in E^{*}$ we have by Hölder inequality with $p>1 / \alpha$, in view $q(\alpha-1)>-1$, that

$$
\begin{aligned}
\mid \varphi & \left(\mathfrak{J}^{\alpha} x(t)-\mathfrak{J}^{\alpha} x(\tau)\right) \mid \Gamma(\alpha) \\
& =\left|\int_{0}^{t}(t-s)^{\alpha-1} \varphi x(s) d s-\int_{0}^{\tau}(\tau-s)^{\alpha-1} \varphi x(s) d s\right| \\
& \leq\left(\int_{0}^{\tau}\left|(t-s)^{\alpha-1}-(\tau-s)^{\alpha-1}\right||\varphi x(s)| d s\right. \\
& \left.+\int_{\tau}^{t}(t-s)^{\alpha-1}|\varphi x(s)| d s\right) \\
& =\left[\left(\int_{0}^{\tau}\left|(t-s)^{\alpha-1}-(\tau-s)^{\alpha-1}\right|^{q} d s\right)^{1 / q}\right. \\
& \left.+\left(\int_{\tau}^{t}(t-s)^{(\alpha-1) q} d s\right)^{1 / q}\right]\|\varphi x\|_{L_{p}[I]} \\
& \leq\left[\left(\int_{0}^{\tau}\left|(t-s)^{q(\alpha-1)}-(\tau-s)^{q(\alpha-1)}\right| d s\right)^{1 / q}\right. \\
& \left.+\left(\frac{(t-\tau)^{(\alpha-1) q+1}}{(\alpha-1) q+1}\right)^{1 / q}\right]\|\varphi x\|_{L_{p}[I]}
\end{aligned}
$$

$$
\begin{aligned}
& =\left[\left(\int_{0}^{\tau}\left|(t-s)^{q(\alpha-1)}-(\tau-s)^{q(\alpha-1)}\right| d s\right)^{1 / q}\right. \\
& \left.+\frac{(t-\tau)^{\alpha-1 / p}}{[q(\alpha-1)+1]^{1 / q}}\right]\|\varphi x\|_{L_{p}[I]} .
\end{aligned}
$$

By noting that $(\alpha-1) q \in(-1,0)$ when $\alpha \in(0,1)$, it can be easily seen that

$$
\begin{gathered}
\int_{0}^{\tau}\left|(t-s)^{q(\alpha-1)}-(\tau-s)^{q(\alpha-1)}\right| d s \\
\leq \frac{1}{q(\alpha-1)+1}(t-\tau)^{q(\alpha-1)+1} .
\end{gathered}
$$

That is,

$$
\begin{aligned}
& \left(\int_{0}^{\tau}\left|(t-s)^{q(\alpha-1)}-(\tau-s)^{q(\alpha-1)}\right| d s\right)^{1 / q} \\
& \quad \leq \frac{(t-\tau)^{\alpha-1 / p}}{[q(\alpha-1)+1]^{1 / q}} .
\end{aligned}
$$

A combination of these results yields

$$
\begin{aligned}
\mid \varphi & \left(\mathfrak{J}^{\alpha} x(t)\right)-\varphi\left(\mathfrak{J}^{\alpha} x(\tau)\right) \mid \\
& =\left|\varphi\left(\mathfrak{J}^{\alpha} x(t)-\mathfrak{J}^{\alpha} x(\tau)\right)\right| \\
& \leq \frac{\|\varphi x\|_{L_{p}[I]}}{\Gamma(\alpha)} \frac{2(t-\tau)^{\alpha-1 / p}}{[q(\alpha-1)+1]^{1 / q}} .
\end{aligned}
$$

Thus, $\mathfrak{\Im}^{\alpha} x \in \mathbf{H}^{\alpha-1 / p}(E)$. This completes the proof.

Corollary 15. For any $\alpha>0, \mathfrak{\Im}^{\alpha}: C\left[I, E_{\omega}\right] \rightarrow C\left[I, E_{\omega}\right]$.

Proof. Let $x \in C\left[I, E_{\omega}\right]$, then $\varphi x \in L_{\infty}$ holds for every $\varphi \in E^{*}$. By noting that the weak continuity implies a strong measurability ([24] page 73), it follows that $x$ is strongly measurable on $I$. Hence, in view of Proposition 2, $x \in$ $\mathscr{H}_{0}^{\infty}(E)$. Consequently $C\left[I, E_{\omega}\right] \subseteq \mathscr{H}_{0}^{p}(E)$ for every $p \in$ $[1, \infty]$. That is, $\mathfrak{J}^{\alpha}: C\left[I, E_{\omega}\right] \rightarrow C\left[I, E_{\omega}\right]$. Hence, the desired result is obtained.

Example 16. Let $\alpha \in[1 / 2,1)$. Define the function $x:[0,1] \rightarrow$ $c_{0}$ by $x(t)=\left\{n \chi_{(1 /(n+1), 1 / n]}\right\}$. We have (in view of Example 12) that

$$
\begin{aligned}
& \mathfrak{J}^{\alpha} x(t)=\left\{0,0, \ldots, 0, \frac{n_{0}\left(t-1 /\left(n_{0}+1\right)\right)^{\alpha}}{\Gamma(1+\alpha)},\right. \\
& \left.\quad \frac{\left(n_{0}+1\right)}{\Gamma(1+\alpha)}\left[\left(t-\frac{1}{n_{0}+2}\right)^{\alpha}-\left(t-\frac{1}{n_{0}+1}\right)^{\alpha}\right], \ldots\right\} .
\end{aligned}
$$

To show that $\mathfrak{\Im}^{\alpha} x$ is norm continuous on $[0,1]$, let $\tau, t \in$ $[0,1]$. With no loss of generality, we may assume that $t, \tau \in$ $I_{n_{0}}$, for some $n_{0} \in \mathbb{N}$. Since the nonzero terms of the sequence in (50) are nonincreasing, then, in view of

$$
|t-\tau| \leq\left(\frac{1}{n_{0}}-\frac{1}{n_{0}+1}\right)=\frac{1}{n_{0}\left(n_{0}+1\right)} \leq \frac{1}{n_{0}^{2}},
$$


we have

$$
\begin{aligned}
& \left\|\mathfrak{J}^{\alpha} x(t)-\mathfrak{J}_{1}^{\alpha} x(\tau)\right\|_{c_{0}} \\
& \quad=\frac{n_{0}}{\Gamma(1+\alpha)}\left|\left(t-\frac{1}{n+1}\right)^{\alpha}-\left(\tau-\frac{1}{n+1}\right)^{\alpha}\right| \\
& \quad \leq \frac{n_{0}}{\Gamma(1+\alpha)}\left|\left(t-\frac{1}{n+1}\right)-\left(\tau-\frac{1}{n+1}\right)\right|^{\alpha} \\
& \quad \leq \frac{n_{0}}{\Gamma(1+\alpha)}|t-\tau|^{\alpha} \leq \frac{1}{\Gamma(1+\alpha)} \frac{|t-\tau|^{\alpha}}{\sqrt{|t-\tau|}} \\
& \quad=\frac{|t-\tau|^{\alpha-1 / 2}}{\Gamma(1+\alpha)} .
\end{aligned}
$$

Thus, the RFPI of $x$ is norm continuous on $[0,1]$. Precisely, since $0<\alpha-1 / 2<\alpha-1 / p<1$, for any $p \leq 2$, then $\mathfrak{J}^{\alpha} x \in \mathbf{H}^{\alpha-1 / 2}(E) \subset \mathbf{H}^{\alpha-1 / p}(E)$. This is precisely what one would expect from Lemma 14 (part (c)).

Analogously, an explicit calculation reveals that $\mathfrak{J}^{\alpha} x$ lies in $C\left([0,1], L_{\infty}[0,1]\right)$, where $x:[0,1] \rightarrow L_{\infty}[0,1]$ defined by Example 10.

We now consider additional mapping properties of the operator $\mathfrak{J}^{\alpha}$. Precisely, we will show that the RFPI enjoys the following commutative property which is folklore in case $E=\mathbb{R}$. However, the proof is completely similar to that of [8], Lemma 3.5.

Lemma 17. Let $\alpha, \beta>0$ and $p>\max \{1,1 / \alpha, 1 / \beta\}$. Then

$$
\mathfrak{I}^{\alpha} \mathfrak{J}^{\beta} x=\mathfrak{I}^{\beta} \mathfrak{J}^{\alpha} x=\mathfrak{J}^{\alpha+\beta} x
$$

holds for every $x \in \mathscr{H}_{0}^{p}(E)$. If $E$ is reflexive, this is also true for every $p \geq 1$.

Proof. Since $p>\max \{1,1 / \alpha, 1 / \beta\}$, it follows that $p>$ $\max \{1,1 /(\alpha+\beta)\}$. So, by Lemmas 8 and 14 , the mappings $\Im^{\alpha} x$, $\mathfrak{\Im}^{\beta} x$, and $\mathfrak{\Im}^{\alpha+\beta} x$ belong to $\mathscr{H}_{0}^{\infty}(E)$ for every $x \in \mathscr{H}_{0}^{p}(E)$. Now repeating the same process as in ([8], Lemma 3.5) implies the claim.

When $E$ is reflexive, the result follows as a direct application of Lemma 14.

\section{Fractional Derivatives of Vector-Valued Functions}

After the notation of the fractional integrals of vectorvalued functions, the fractional derivatives become a natural requirement. Before we come to the definitions and a detailed study of the mathematical properties of fractional differential operators, we recall the following.

Definition 18. Consider the vector-valued function $x: I \rightarrow$ E:

(1) Let $\varphi x$ be differentiable on $I$ for every $\varphi \in E^{*}$. The function $x$ is said to be weakly differentiable on $I$ if there exists $y: I \rightarrow E$ such that for every $\varphi \in E^{*}$ we have

$$
\frac{d \varphi x(t)}{d t}=\varphi y(t), \quad \text { for every } t \in I .
$$

The function $y$ is called the weak derivative of the function $x$.

(2) Let $\varphi x$ be differentiable a.e. on $I$ for every $\varphi \in E^{*}$ (the null set may vary with $\varphi \in E^{*}$ ). The function $x$ is said to be pseudo differentiable on $I$ if there exists a function $y: I \rightarrow E$ such that for every $\varphi \in E^{*}$ there exists a null set $N(\varphi) \subset I$ such that

$$
(\varphi x(t))^{\prime}=\varphi y(t), \quad \text { for every } t \in \frac{I}{N(\varphi)} .
$$

In this case, the function $y$ is called the pseudo derivative of $x$.

If $x$ is pseudo differentiable on $I$ and the null set invariant for every $\varphi \in E^{*}$, then $x$ is a.e. weakly differentiable on $I$.

Clearly, if $x$ is a.e. weakly differentiable on $I$, then $\varphi(x)$ is a.e. differentiable on $I$. The converse holds in a weakly sequentially complete space (see [25], Theorem 7.3.3).

For more details of the derivatives of vector-valued functions we refer to $[10,12,26]$.

The following results play a major role in our analysis

Proposition 19 (see [27], Theorem 5.1). The function $y: I \rightarrow$ $E$ is an indefinite Pettis integrable, if and only if $y$ is weakly absolutely continuous on I and have a pseudo derivative on I. In this case, $y$ is an indefinite Pettis integral of any of its pseudo derivatives.

Now we are in the position to define the fractional-type derivatives of vector-valued functions.

Definition 20. Let $x: I \rightarrow E$. For the positive integer $m$ such that $\alpha \in(m-1, m), m \in \mathbb{N}_{0}:=\{0,1,2, \ldots\}$ we define the Caputo fractional-pseudo (weak) derivative "shortly CFPD (CFWD)" of $x$ of order $\alpha$ by

$$
\frac{d^{\alpha}}{d t^{\alpha}} x(t):=\mathfrak{J}^{m-\alpha} D^{m} x(t),
$$

where the sign " $D$ " denotes the pseudo (or weak) differential operator. We use the notation $d_{p}^{\alpha} / d t^{\alpha}$ and $d_{\omega}^{\alpha} / d t^{\alpha}$ to characterize the Caputo fractional-pseudo derivatives and Caputo fractional weak derivatives, respectively.

It is well known that, although the weak derivative of a weakly differentiable function is uniquely determined, the pseudo derivative of the pseudo differentiable function is not unique. Also, although any two pseudo derivatives $y, z$ of a function $x: I \rightarrow E$ need not be a.e. equal (see [12, Example 9.1] and [26, p. 2]), the functions $y, z$ are weakly equivalent on $I$ (that is, $\varphi y=\varphi z$ holds a.e. for every $\varphi \in E^{*}$ ). The 
next lemma provides a useful characterization property of the CFPD. Really, it can be easily seen that the CFPD of a Caputo fractional-pseudo differentiable function does not depend on the choice of a pseudo derivative of the function.

Lemma 21. Let $x: I \rightarrow E$ be pseudo differentiable function where pseudo derivatives lie in $\mathscr{H}_{0}^{p}(E)$. If $\left(d_{p}^{\alpha} / d t^{\alpha}\right) x$ exists on $I$, then the CFPD of $x$ depends on the choice of the pseudo derivatives of $x$.

Proof. Let $y, z \in \mathscr{H}_{0}^{p}(E)$ be two pseudo derivatives of the pseudo differentiable function $x$. Since $x$ and $y$ are weakly equivalent on $I$, then for every $\varphi \in E^{*}$ there exists a null set $\mathfrak{N}$ which depends on $\varphi \in E^{*}$ such that $(t-s)^{-\alpha} \varphi y(s)=$ $(t-s)^{-\alpha} \varphi z(s)$ for every $s \in[0, t] / \mathfrak{N}$. Consequently, for almost every $t \in I$,

$$
\begin{aligned}
\varphi\left(\mathfrak{\Im}^{1-\alpha} y(t)\right) & =\mathfrak{\Im}^{1-\alpha} \varphi y(t)=\mathfrak{\Im}^{1-\alpha} \varphi z(t) \\
& =\varphi\left(\mathfrak{\Im}^{1-\alpha} z(t)\right)
\end{aligned}
$$

holds for every $\varphi \in E^{*}$. Thus,

$$
\frac{d_{p}^{\alpha}}{d t^{\alpha}} x=\mathfrak{\Im}^{1-\alpha} D_{p} x=\mathfrak{\Im}^{1-\alpha} y=\mathfrak{\Im}^{1-\alpha} z \text { on } I,
$$

which is what we wished to show.

We consider the following examples.

Example 22. Define $x:[0,1] \rightarrow \ell_{2}$ by

$$
x(t):=\left\{\ln \left(1+\frac{t}{n}\right)\right\}, \quad n \in \mathbb{N} .
$$

We claim that the CFWD of $x$ exists on $[0,1]$. To see this, we let $\varphi \in\left(\ell_{2}\right)^{*}=\ell_{2}$. Then there corresponds to $\varphi$ a sequence $\left\{\lambda_{n}\right\} \in \ell_{2}$ such that

$$
\varphi x(t)=\sum_{n} \lambda_{n} \ln \left(1+\frac{t}{n}\right) .
$$

Since the series in (60) converges uniformly on $[0,1]$, the formal differentiation of $\varphi x$ yields

$$
(\varphi x(t))^{\prime}=\sum_{n} \frac{\lambda_{n}}{n+t}=\varphi\left(\left\{\frac{1}{t+n}\right\}\right) .
$$

It is not hard to justify the differentiation by noting that the series in the right hand side of (61) is uniformly and absolutely convergent on $[0,1]$. Consequently, $\varphi x$ is differentiable on $[0,1]$ for $\varphi \in\left(\ell_{2}\right)^{*}$, meaning that $x$ is weakly differentiable on $[0,1]$ (because $\ell_{2}$ is weakly complete (cf. [25], Theorem 7.3.3) and $D_{\omega} x(t)=\{1 /(t+n)\}$. Owing to Lemma 25, the CFWD of any order $\alpha \in(0,1)$ of $x$ exists on $[0,1]$. To calculate the CFWD of $x$ on $[0,1]$ we observe, in view of Example 11, that

$$
\begin{aligned}
\frac{d_{\omega}^{\alpha}}{d t^{\alpha}} x(t) & =\mathfrak{\Im}^{1-\alpha} D_{\omega} x(t) \\
& =\left\{\frac{t^{1-\alpha}}{n \Gamma(2-\alpha)}{ }_{2} \mathrm{~F}_{1}\left(1,1,2-\alpha, \frac{-t}{n}\right)\right\} .
\end{aligned}
$$

Example 23. Let $E$ be an infinite dimension Banach space that fails cotype. Define $y:[0,1] \rightarrow E$ by $y(t):=\int_{0}^{t} x(s) d s$, where $x:[0,1] \rightarrow E$ given by formula (7). As cited in ([13], Corollary 4$), y$ is nowhere weakly differentiable on $[0,1]$. Consequently the CFWD of any order $\alpha \in[3 / 4,1)$ of $y$ loses its meaning on $[0,1]$.

Remark 24. As shown in Example 23, there is an infinite dimension Banach space $E$ and weakly absolutely continuous function $y: I \rightarrow E$ which is nowhere weakly differentiable (hence the CFWD of $y$ does not exist). Also, even when $E$ is separable, and $y$ is Lipschitz function, the pseudo derivatives (hence the CFPD) of $y$ need not to exist [28].

However, Definition 20 of the CFPD (CFWD) has the disadvantage that it completely loses its meaning if the function $x$ fails to be pseudo (weakly) differentiable. Precisely, the CFPD (in particular the CFWD) of a function $x$ loses its meaning if $x$ is not weakly absolutely continuous.

The next lemma gives sufficient conditions that ensure the existence of the Caputo fractional derivatives of a function $x \in \mathscr{H}^{p}(E)$.

Lemma 25. Let $\alpha \in(0,1)$. For the function $x: I \rightarrow E$, the following hold:

(a) If $x$ has a pseudo derivative $D_{p} x \in \mathscr{H}_{0}^{p}(E)$, where $p>$ $1 /(1-\alpha)$, then the CFPD of $x$ of order $\alpha$ exists on $I$. Moreover, $\left(d_{p}^{\alpha} / d t^{\alpha}\right) x \in C\left[I, E_{\omega}\right]$.

(b) If $E$ is weakly complete or contains no copy of $c_{0}$ and if $x$ has a weak derivative $D_{\omega} x \in \mathscr{H}^{p}(E)$, where $p \in$ $[1, \infty]$, then the CFWD of $x$ of order $\alpha$ exists on $I$. Moreover, $\left(d_{\omega}^{\alpha} / d t^{\alpha}\right) x \in C\left[I, E_{\omega}\right]$.

This holds for any $x \in \mathscr{H}^{p}(E)$ with $p \in[1, \infty]$ if $E$ is reflexive.

In all cases, $\varphi\left(\left(d_{p}^{\alpha} / d t^{\alpha}\right) x\right)=\left(d_{p}^{\alpha} / d t^{\alpha}\right) \varphi x\left(\varphi\left(\left(d_{\omega}^{\alpha} / d t^{\alpha}\right) x\right)=\right.$ $\left.\left(d_{\omega}^{\alpha} / d t^{\alpha}\right) \varphi x\right)$ holds for every $\varphi \in E^{*}$.

Proof. Since the weak (pseudo) derivative of an a.e. weakly (pseudo) differentiable function is strongly (weakly) measurable $[12,26,28]$, the proof is readily available, in view of the definition of Caputo fractional derivatives and Lemma 8. Moreover, since $D_{\omega} x\left(D_{p} x\right) \in \mathscr{H}_{0}^{p}(E)$ with $p>1 /(1-\alpha)$, it follows, in view of Lemma 14 , that $\mathfrak{\Im}^{1-\alpha} D_{\omega} x\left(\mathfrak{\Im}^{1-\alpha} D_{p} x\right) \epsilon$ $C\left[I, E_{\omega}\right]$. This completes the proof.

Besides the Caputo fractional-pseudo (weak) derivatives, we define the Riemann-Liouville fractional-pseudo (weak) derivatives.

Definition 26. Let $x: I \rightarrow E$. For the positive integer $m$ such that $\alpha \in(m-1, m), m \in \mathbb{N}_{0}:=\{0,1,2, \ldots\}$ we define the Riemann-Liouville fractional-pseudo (weak) derivative "shortly RFPD (RFWD)" of $x$ of order $\alpha$ by

$$
\mathfrak{D}^{\alpha} x(t):=D^{m} \mathfrak{J}^{m-\alpha} x(t),
$$

where $D$ is defined as in Definition 20. We use the notation $\mathfrak{D}_{p}^{\alpha}\left(\mathfrak{D}_{\omega}^{\alpha}\right)$ to characterize the Riemann-Liouville fractionalpseudo (weak) derivatives. 
Clearly, in infinite dimension Banach spaces, the weak absolute continuity of $\mathfrak{J}^{m-\alpha} x$, is necessarily (but not sufficient) condition for the existence of RFPD (in particular RFWD) of $x$.

Lemma 27. Let $0<\alpha<1$. For any $x \in \mathscr{H}_{0}^{p}(E)$ with $p>$ $\max \{1 / \alpha, 1 /(1-\alpha)\}$, we have

$$
\mathfrak{D}_{p}^{\alpha} \mathfrak{\Im}^{\alpha} x=x \text { a.e. }
$$

If $E$ is reflexive, this is also true for every $p \geq 1$.

Proof. Our assumption $x \in \mathscr{H}_{0}^{p}(E)$ with $p>\max \{1 / \alpha, 1 /(1-$ $\alpha)\}$ yields, in view of Lemma 17, that

$$
\mathfrak{D}_{p}^{\alpha} \mathfrak{\Im}^{\alpha} x=D_{p} \mathfrak{I}^{1-\alpha} \mathfrak{J}^{\alpha} x=D_{p} \mathfrak{I}^{1} x .
$$

The claim now follows immediately, since (cf. Proposition 19) the indefinite integral of Pettis integrable function is weakly absolutely continuous and it is pseudo differentiable with respect to the right endpoint of the integration interval and its pseudo derivative equals the integrand at that point.

Remark 28. When we replace $\mathfrak{D}_{p}^{\alpha}$ by $\mathfrak{D}_{\omega}^{\alpha}$, then Lemma 27 is no longer necessarily true for arbitrary $x \in \mathscr{H}_{0}^{1}(E)$ even when $E$ is reflexive: evidently, in [13, remark below Corollary 4] the existence of a reflexive Banach space $E$ and a strong measurable Pettis integrable function $x: I \rightarrow E$ was proved such that $x$ has nowhere weakly differentiable integral. In this case, $D_{\omega} \mathfrak{I}^{1} x$ lost its meaning. This gives a reason to believe that (65) (hence (64)) with $\mathfrak{D}_{\omega}^{\alpha} x$ could not happen.

However, we have the following result.

Lemma 29. Let $0<\alpha<1$. For any $x \in C\left[I, E_{\omega}\right]$, we have

$$
\mathfrak{D}_{\omega}^{\alpha} \mathfrak{\Im}^{\alpha} x=x \text { on } I \text {. }
$$

Proof. Our assumption $x \in C\left[I, E_{\omega}\right] \subset \mathscr{H}_{0}^{\infty}(E)$ (see the proof of Corollary 15) yields, in view of Lemma 17, that

$$
\mathfrak{D}_{\omega}^{\alpha} \mathfrak{\Im}^{\alpha} x=D_{\omega} \mathfrak{I}^{1-\alpha} \mathfrak{J}^{\alpha} x=D_{\omega} \mathfrak{\Im}^{1} x .
$$

The claim now follows immediately, since the indefinite integral of weakly contentious function is weakly absolutely continuous and it is weakly differentiable with respect to the right endpoint of the integration interval and its weak derivative equals the integrand at that point.

The following lemma is folklore in case $E=\mathbb{R}$, but to see that it also holds in the vector-valued case, we provide a proof.

Lemma 30. Let $0<\alpha<1$ and $p>\max \{1 / \alpha, 1 /(1-\alpha)\}$. If the function $x: I \rightarrow E$ is weakly absolutely continuous on $I$ and passes a pseudo derivative in $\mathscr{H}_{0}^{p}(E)$, then

$$
\frac{d_{p}^{\alpha} x}{d t^{\alpha}}(t)=\mathfrak{D}_{p}^{\alpha} x(t)-\frac{t^{\alpha}}{\Gamma(1+\alpha)} x(0) .
$$

In particular, if $x$ passes a weak derivative in $C\left[I, E_{\omega}\right]$, then

$$
\frac{d_{\omega}^{\alpha} x}{d t^{\alpha}}(t)=\mathfrak{D}_{\omega}^{\alpha} x(t)-\frac{t^{\alpha}}{\Gamma(1+\alpha)} x(0) .
$$

If $E$ is reflexive, this is also true for every $p \geq 1$.
Proof. We observe that, under the assumption imposed on $D_{p} x$ together with Proposition 3, the weakly absolutely continuity of $x$ is equivalent to

$$
x(t)=x(0)+\int_{0}^{t} D_{p} x(s) d s .
$$

Hence, owing to Lemma 27, it follows that

$$
\begin{aligned}
\frac{d_{p}^{\alpha} x}{d t^{\alpha}} & =\mathfrak{J}^{1-\alpha} D_{p} x=\left(\mathfrak{D}_{p}^{\alpha} \mathfrak{J}^{\alpha}\right) \mathfrak{\Im}^{1-\alpha} D_{p} x \\
& =\mathfrak{D}_{p}^{\alpha}\left(\mathfrak{J}^{\alpha} \mathfrak{J}^{1-\alpha}\right) D_{p} x=\left(D_{p} \mathfrak{\Im}^{1-\alpha}\right) \mathfrak{I}^{1} D_{p} x \\
& =D_{p} \mathfrak{J}^{1-\alpha}(x(t)-x(0)) \\
& =\mathfrak{D}_{p}^{\alpha} x(t)-\frac{t^{\alpha}}{\Gamma(1+\alpha)} x(0)
\end{aligned}
$$

which is what we wished to show.

The proof of (69) is very similar to that in (68); therefore, we omit the details.

Remark 31. As we remark above, the definition of the CFPD of a function $x$ loses completely its meaning if $x$ is not weakly absolutely continuous. For this reason, we are able to use Lemma 30 to define the Caputo fractional derivative in general; that is, we put

$$
\frac{d_{p}^{\alpha} x}{d t^{\alpha}}(t):=\mathfrak{D}_{p}^{\alpha} x(t)-\frac{t^{\alpha}}{\Gamma(1+\alpha)} x(0), \quad 0<\alpha<1 .
$$

Similarly, we define

$$
\frac{d_{\omega}^{\alpha} x}{d t^{\alpha}}(t):=\mathfrak{D}_{\omega}^{\alpha} x(t)-\frac{t^{\alpha}}{\Gamma(1+\alpha)} x(0) .
$$

However, Lemma 30 claims that, for the weakly absolutely continuous functions having Pettis integrable pseudo [weak] derivatives, definitions (72) and (73) of the Caputo fractionalpseudo [weak] derivatives coincide with Definition 20.

\section{An Application}

Let $f: I \times E \rightarrow E$ be given function. Consider the boundary value problem of the fractional type

$$
\begin{aligned}
\frac{d_{\omega}^{\alpha}}{d t^{\alpha}} y(t) & =\lambda f(t, y(t)), \\
\alpha(0)+b y(1) & =h,
\end{aligned}
$$

with certain constants $b, h \in \mathbb{R}, b \neq-1$. To obtain the integral equation modeled off the problem (74), we let $y$ be a weakly continuous solution to problem (74); then formally we have

$$
\mathfrak{\Im}^{\alpha} \frac{d_{\omega}^{\alpha}}{d t^{\alpha}} y(t)=\lambda \mathfrak{\Im}^{\alpha} f(t, y(t))
$$


that is

$$
\begin{aligned}
\mathfrak{J}^{\alpha} \mathfrak{J}^{1-\alpha} D_{\omega} y(t) & =\lambda \mathfrak{\Im}^{\alpha} f(t, y(t)) \Longrightarrow \\
\mathfrak{I}^{1} D_{\omega} y(t) & =\lambda \mathfrak{J}^{\alpha} f(t, y(t)) .
\end{aligned}
$$

This reads (cf. [29])

$$
\begin{aligned}
y(t)=y(0)+\lambda \Im^{\alpha} f(t, y(t)), & \\
\alpha & \in(0,1), t \in I, \lambda \in \mathbb{R},
\end{aligned}
$$

with some (presently unknown) quantity $y(0)$.

Now, we solve (77) for $y(0)$ by $y(0)+b y(1)=h$, and it follows that

$$
(1+b) y(0)+\frac{\lambda b}{\Gamma(\alpha)} \int_{0}^{1}(1-s)^{\alpha-1} f(s, y(s)) d s=h .
$$

Thus,

$$
y(0)=\frac{h}{1+b}-\frac{\lambda b}{1+b} I^{\alpha} f(1, y(1)) .
$$

Assume that the function $f$ is weakly-weakly continuous function such that

(1) for any $r>0$, there is a constant $M>0$ such that $\|f(t, y)\| \leq M$ for all $t \in I$ and $\|y\| \leq r$,

(2) there exists a nondecreasing continuous function $\psi$ : $[0, \infty) \rightarrow[0, \infty), \psi(0)=0$ and $\psi(t)<t$ for all $t>0$ such that $\beta(f(T \times A)) \leq \psi(\beta(A))$ for every bounded set $A \subset E$, where $\beta$ stands for De Blasi's weak measure of noncompactness (see [30]).

Occasionally, if $f: I \times E \rightarrow E$ is weakly-weakly continuous and $E$ is reflexive, then the assumptions (1) and (2) are automatically satisfied (see, e.g., [31]).

Theorem 32 (see [6], Theorem 3.3). Let $\alpha \in(0,1)$ and $|\lambda|<$ $\Gamma(1+\alpha)$. If $f: I \times E \rightarrow E$ is weakly-weakly continuous and satisfies the assumptions (1) and (2), then the integral equation (77) has a weakly continuous solution $y$ defined on $[0,1]$.

Proof. We omit the proof since it is almost identical to that in the proof in ([6], Theorem 3.3) with (small) necessary changes.

In the following example we assume that $y \in C\left[I, E_{\omega}\right]$ solves (77) and we will show that, not only do we have that $y$ no longer necessarily solves (74) (when the Caputo fractional weak derivative is taken in the sense of Definition 20), but even worse, it could happen that the problem (74) is "meaningless" on $I$.

Example 33. Let $\alpha \in(0,1 / 4]$. Let $E$ be an indefinite dimensional reflexive Banach space that fails cotype. Define the weakly-weakly continuous function $f:[0,1] \times E \rightarrow E$ by $f:=\mathfrak{J}^{1-\alpha} x$, where $x: I \rightarrow E$ is defined by formula (7). Obviously (cf. Example 6, in view of Remark 7), $f$ satisfies the assumptions of Theorem 32.
Now, consider the integral equation (77) with $f=\mathfrak{J}^{1-\alpha} x$. Namely, we consider the integral equation

$$
\begin{aligned}
y(t)=y(0)+\lambda \mathfrak{\Im}^{\alpha} \mathfrak{J}^{1-\alpha} x(t), & \\
\alpha & \in\left(0, \frac{1}{4}\right], t \in I, \lambda \in \mathbb{R} .
\end{aligned}
$$

Obviously, the solution $y$ is weakly continuous on $[0,1]$ (this is an immediate consequence of Remark 7 and Lemma 17).

Since $\varphi x \in L_{1}[0,1]$ holds for every $\varphi \in E^{*}$, it follows by the commutative property of the fractional integral operators calculus over the space of Lebesgue integrable functions (see [21], Section 2.3) that

$$
\begin{aligned}
\varphi\left(\mathfrak{J}^{\alpha} \mathfrak{J}^{1-\alpha} x(t)\right) & =\mathfrak{J}^{\alpha} \varphi\left(\mathfrak{J}^{1-\alpha} x(t)\right) \\
& =\mathfrak{J}^{\alpha} \mathfrak{I}^{1-\alpha} \varphi(x(t))=\mathfrak{I}^{1} \varphi(x(t)) \\
& =\varphi\left(\mathfrak{J}^{1} x(t)\right),
\end{aligned}
$$

that is

$$
\varphi\left(\mathfrak{J}^{\alpha} \mathfrak{I}^{1-\alpha} x(t)-\mathfrak{I}^{1} x(t)\right)=0, \quad \text { for every } \varphi \in E^{*} .
$$

Hence, $\mathfrak{J}^{\alpha} \mathfrak{J}^{1-\alpha} x=\mathfrak{I}^{1} x$. Consequently, problem (80) becomes

$$
y(t)=y(0)+\lambda \mathfrak{\Im}^{1} x(t), \quad \alpha \in\left(0, \frac{1}{4}\right], t \in I .
$$

As showed in ([13], Corollary 4), the indefinite integral $t \rightarrow$ $\int_{0}^{t} x(s) d s$ is nowhere weakly differentiable on $[0,1]$. Thus, $y$ is nowhere weakly differentiable on $[0,1]$; hence the CFWD of $y$ (hence the boundary value problem) completely loses its meaning if the CFWD is taken in the sense of Definition 20. This is what we wished to show.

Now, we are in the position to state and prove the following existence theorem.

Theorem 34. If $f: I \times E \rightarrow E$ is a function such that all conditions from Theorem 32 hold, then problem (74) (where the Caputo fractional weak derivative is taken in the sense (73)) has a weak solution on $[0,1]$.

Proof. Let $y \in C\left[I, E_{\omega}\right]$ be a solution to (77). Equip $E$ and $I \times E$ with weak topology and note that $t \mapsto(t, y(t))$ is continuous as a mapping from $I$ into $I \times E$. Since $f: I \times E \rightarrow$ $E$ is weakly-weakly continuous on $[0,1]$, then $f(\cdot, y(\cdot))$ is a composition of this mapping with $f$ and thus $f(\cdot, y(\cdot))$ is weakly continuous on $[0,1]$.

Now, operating by the operator $\mathfrak{D}_{\omega}^{\alpha}$ on both sides of (77), it follows by Lemma 29 that

$$
\mathfrak{D}_{\omega}^{\alpha} y=\mathfrak{D}_{\omega}^{\alpha} y(0)+\lambda \mathfrak{D}_{\omega}^{\alpha} \mathfrak{\Im}^{\alpha} f=\frac{t^{-\alpha}}{\Gamma(1-\alpha)} y(0)+\lambda f .
$$

Now, insert definition (73) of the Caputo fractional derivative, and we get

$$
\frac{d_{\omega}^{\alpha}}{d t^{\alpha}} y(t)=\lambda f(t, y(t)) .
$$


With some further efforts, one can get the boundary condition $y(0)+b y(1)=h$.

Therefore, $y$ satisfies problem (74) which is what we wished to show.

\section{Data Availability}

No data were used to support this study

\section{Conflicts of Interest}

The author declares no conflicts of interest.

\section{References}

[1] H. A. Salem and A. M. El-Sayed, "Weak solution for fractional order integral equations in reflexive Banach spaces," Mathematica Slovaca, vol. 55, no. 2, pp. 169-181, 2005.

[2] H. A. Salem, A. M. El-Sayed, and O. L. Moustafa, "A note on the fractional calculus in Banach spaces," Studia Scientiarum Mathematicarum Hungarica, vol. 42, no. 2, pp. 115-130, 2005.

[3] R. P. Agarwal, V. Lupulescu, D. O’Regan, and G. ur Rahman, "Multi-term fractional differential equations in a nonreflexive Banach space," Advances in Difference Equations, 2013:302, 18 pages, 2013.

[4] R. P. Agarwal, V. Lupulescu, D. O’Regan, and G. ur Rahman, "Fractional calculus and fractional differential equations in nonreflexive Banach spaces," Communications in Nonlinear Science and Numerical Simulation, vol. 20, no. 1, pp. 59-73, 2015.

[5] R. P. Agarwal, V. Lupulescu, D. O’Regan, and G. ur Rahman, "Nonlinear fractional differential equations in nonreflexive Banach spaces and fractional calculus," Advances in Difference Equations, 2015:112, 18 pages, 2015.

[6] R. P. Agarwal, V. Lupulescu, D. O’Regan, and G. Rahman, "Weak solutions for fractional differential equations in nonreflexive Banach spaces via Riemann-Pettis integrals," Mathematische Nachrichten, vol. 289, no. 4, pp. 395-409, 2016.

[7] H. A. Salem, "On the fractional order m-point boundary value problem in reflexive Banach spaces and weak topologies," Journal of Computational and Applied Mathematics, vol. 224, no. 2, pp. 565-572, 2009.

[8] H. A. Salem, "On the fractional calculus in abstract spaces and their applications to the Dirichlet-type problem of fractional order," Computers and Mathematics with Applications, vol. 59, no. 3, pp. 1278-1293, 2010.

[9] H. A. H. Salem and M. Cichoń, "On solutions of fractional order boundary value problems with integral boundary conditions in Banach spaces," journal of function spaces and applications, vol. 2013, Article ID 428094, 2013.

[10] J. Diestel and J. J. Uhl Jr., Vector Measures, vol. 15 of Mathematical Surveys, American Mathematical Society, Providence, RI, USA, 1977.

[11] R. H. Martin and J. J. Uhl Jr., Nonlinear Operators And Differential Equations in Banach Spaces, John Wiley \& Sons, New York, NY, USA, 1976.

[12] B. J. Pettis, "On integration in vector spaces," Transactions of the American Mathematical Society, vol. 44, no. 2, pp. 277-304, 1938.

[13] S. J. Dilworth and M. Girardi, "Nowhere weak differentiability of the Pettis integral," Quaestiones Mathematicae, vol. 18, no. 4, pp. 365-380, 1995.
[14] G. A. Edgar, "Measurability in a Banach space," Indiana University Mathematics Journal, vol. 26, no. 4, pp. 663-677, 1977.

[15] G. A. Edgar, "Measurability in a Banach space, II," Indiana University Mathematics Journal, vol. 28, no. 4, pp. 559-579, 1979.

[16] J. L. Gàmez and J. Mendoza, "On denjoy-dunford and denjoypettis integrals," Studia Mathematica, vol. 130, no. 2, pp. 115-133, 1998.

[17] R. A. Gordon, "The Denjoy extension of the Bochner, Pettis, and DUNford integrals," Studia Mathematica, vol. 92, no. 1, pp. 7391, 1989.

[18] R. F. Geitz, "Pettis integration," Proceedings of the American Mathematical Society, vol. 82, no. 1, pp. 81-86, 1981.

[19] R. F. Geitz, "Geometry and the Pettis integral," Transactions of the American Mathematical Society, vol. 269, no. 2, pp. 535-548, 1982.

[20] A. A. Kilbas, H. M. Srivastava, and J. J. Trujillo, Theory and Applications of Fractional Differential Equations, New York, NY, USA, Elsevier, 2006.

[21] S. G. Samko, A. A. Kilbas, and O. I. Marichev, Fractional Integrals and Derivatives, Theory and Applications, Gordon and Breach, Yverdon, Switzerland, 1993.

[22] M. Mendel and A. Naor, "Metric cotype," Annals of Mathematics: Second Series, vol. 168, no. 1, pp. 247-298, 2008.

[23] B. Ross, S. G. Samko, and E. R. Love, "Functions that have no first order derivative might have fractional derivatives of all orders less than one," Real Analysis Exchange, vol. 20, no. 1, pp. 140-157, 1994/95.

[24] E. Hille and R. S. Phillips, Functional analysis and semi-groups, American Mathematical Society Colloquium Publications, vol. 31, American Mathematical Society, Providence, RI, USA, 1957.

[25] S. Schwabik and Y. Guoju, Topics in Banach Space Integration, World Scientific, Singapore, 2005.

[26] D. W. Solomon, "On differentiability of vector-valued functions of a real variable," Studia Mathematica, vol. 29, pp. 1-4, 1967.

[27] K. Naralenkov, "On Denjoy type extensions of the Pettis integral," Czechoslovak Mathematical Journal, vol. 60(135), no. 3, pp. 737-750, 2010.

[28] D. W. Solomon, Denjoy integration in abstract spaces, Memoris of the American Mathematical Society, No. 85, American Mathematical Society, Providence, R.I., 1969.

[29] A. R. Mitchell and C. Smith, "An existence theorem for weak solutions of differential equations in Banach spaces," in Nonlinear Equations in Abstract Spaces, V. Lakshmikantham, Ed., pp. 387-404, 1978.

[30] F. S. De Blasi, "On a property of the unit sphere in a Banach space," Bulletin Mathematiques De La Societe Des Sciences Mathematiques De Roumanie, vol. 21, no. 3, pp. 259-262, 1977.

[31] A. Szep, "Existence theorem for weak solutions of ordinary differential equations in reflexive Banach spaces," Studia Scientiarum Mathematicarum Hungarica, vol. 6, pp. 197-203, 1971. 


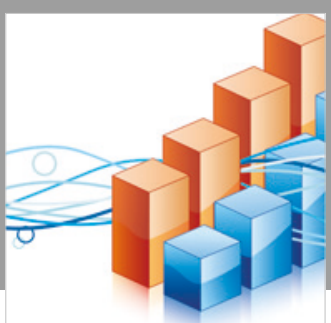

Advances in

Operations Research

\section{-n-m}
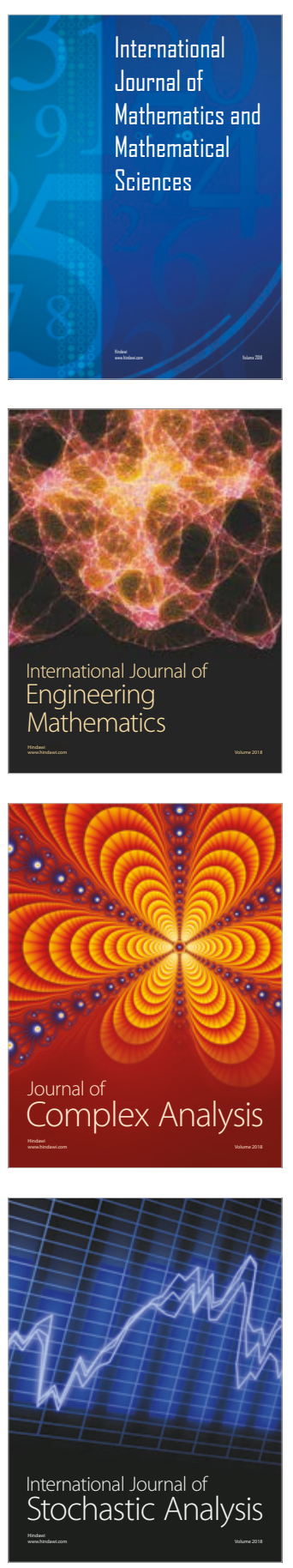
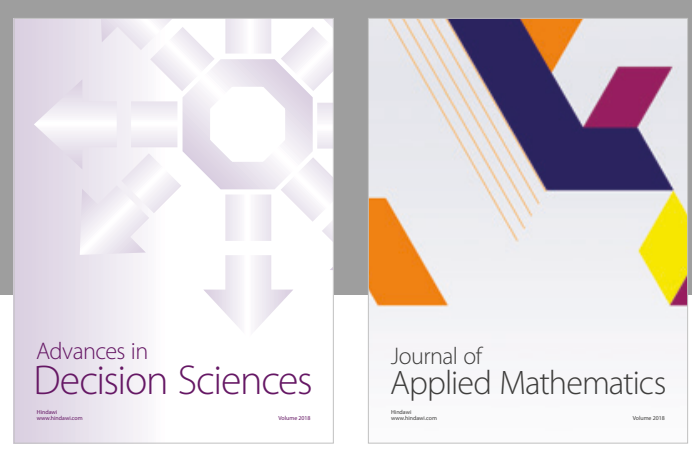

Journal of

Applied Mathematics
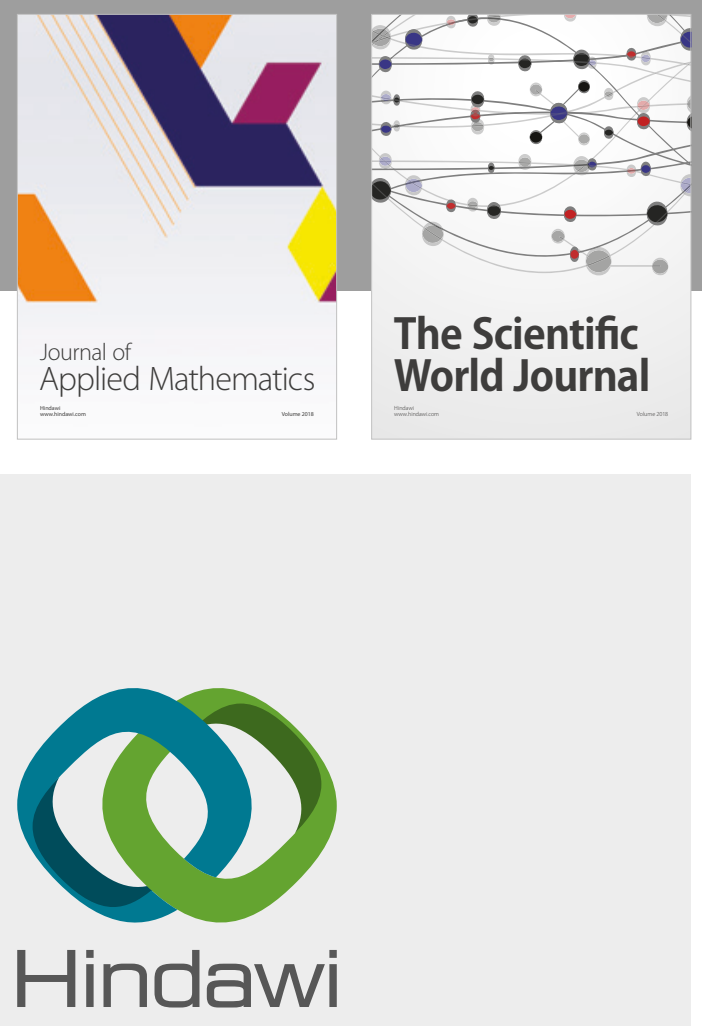

Submit your manuscripts at

www.hindawi.com

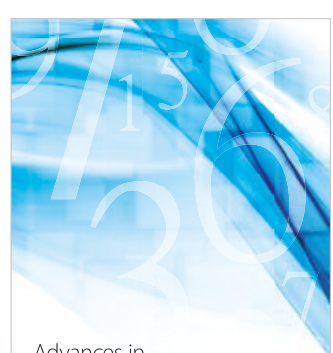

Advances in
Numerical Analysis
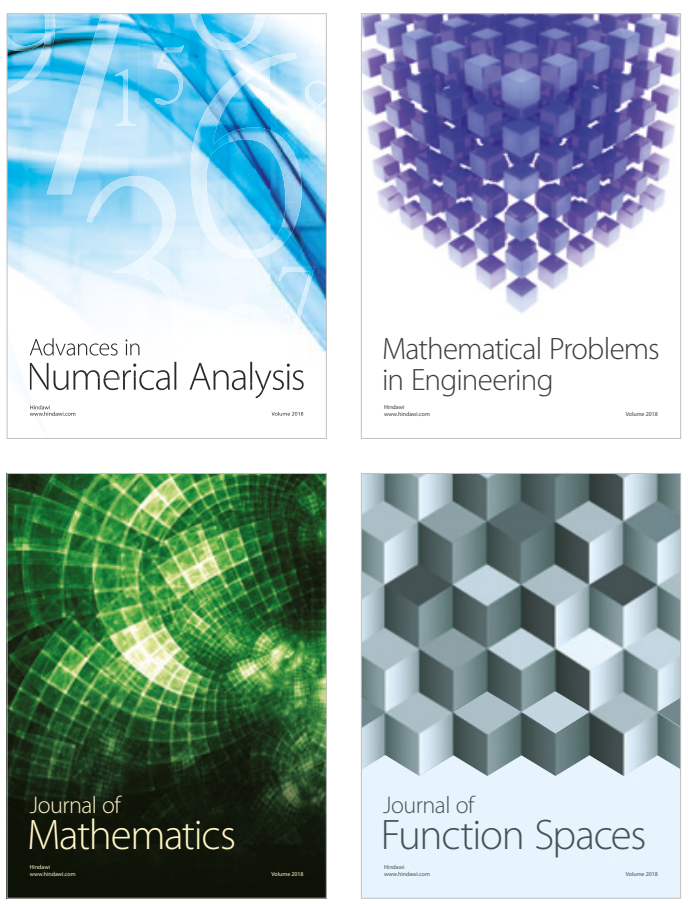

Mathematical Problems in Engineering

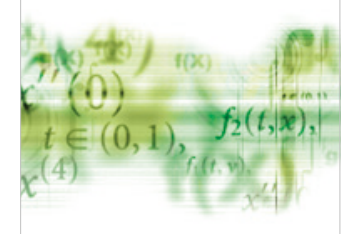

International Journal of

Differential Equations

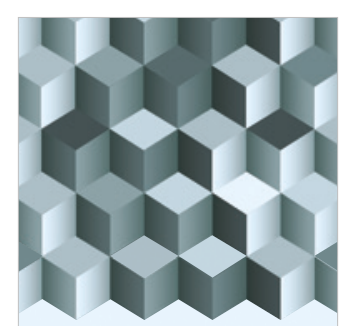

Journal of

Function Spaces

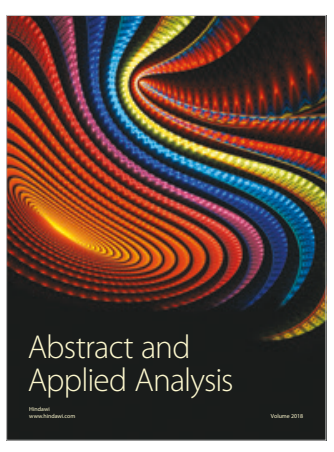

The Scientific

World Journal

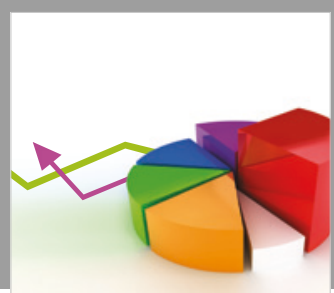

Journal of

Probability and Statistics
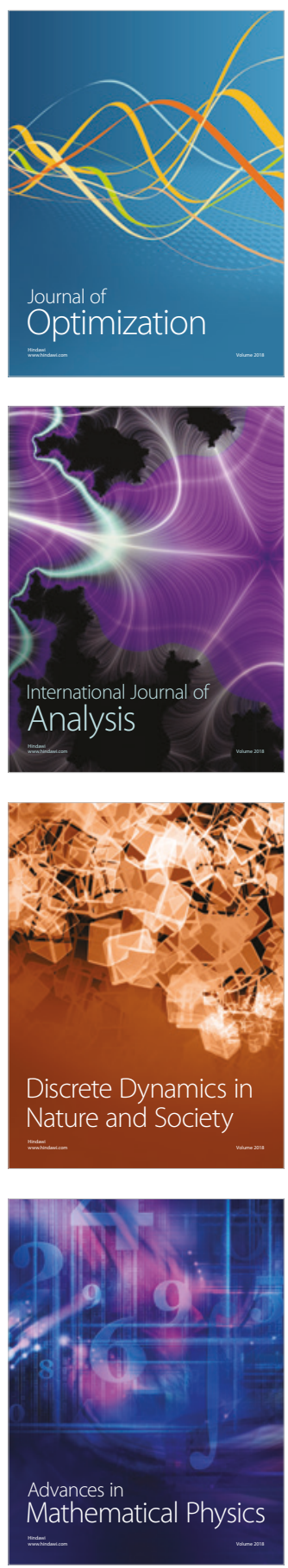